\title{
Solving Multiobjective Mixed Integer Convex Optimization Problems
}

\author{
Marianna de Santis*, Gabriele Eichfelder,* Julia Niebling,, Stefan Rocktäschel ${ }^{* *}$
}

July 28,2020

\begin{abstract}
Multiobjective mixed integer convex optimization refers to mathematical programming problems where more than one convex objective function needs to be optimized simultaneously and some of the variables are constrained to take integer values. We present a branch-and-bound method based on the use of properly defined lower bounds. We do not simply rely on convex relaxations, but we build linear outer approximations of the image set in an adaptive way. We are able to guarantee correctness in terms of detecting both the efficient and the nondominated set of multiobjective mixed integer convex problems according to a prescribed precision. As far as we know, the procedure we present is the first non-scalarization based deterministic algorithm devised to handle this class of problems. Our numerical experiments show results on biobjective and triobjective mixed integer convex instances.
\end{abstract}

Key Words: Multiobjective Optimization, Mixed Integer Convex Programming

Mathematics subject classifications (MSC 2010): 90C11, 90C26, 90C29

\section{Introduction}

Multiobjective programming is concerned with mathematical problems where more than one objective function needs to be optimized simultaneously. When the problem considered involves both continuous and integer variables we are in the context of multiobjective mixed integer programming. In this paper, we focus on multiobjective mixed integer programming problems of the following form:

$$
\begin{array}{cl}
\min & \left(f_{1}(x), \ldots, f_{m}(x)\right)^{T} \\
\mathrm{s.t.} & g_{k}(x) \leq 0 \quad k=1, \ldots, p \\
& x \in B:=[l, u] \\
& x_{i} \in \mathbb{Z} \quad \forall i \in I,
\end{array}
$$

*Department of Computer, Control and Management Engineering, Sapienza Università di Roma, Via Ariosto 2500185 Roma, Italy, mdesantis@diag.uniroma1.it

**Institute for Mathematics, Technische Universität Ilmenau, Po 1005 65, D-98684 Ilmenau, Germany, \{gabriele.eichfelder, julia.niebling, stefan.rocktaeschel\}@tu-ilmenau.de 
where $f_{j}, g_{k}: B \rightarrow \mathbb{R} ; j=1, \ldots, m ; k=1, \ldots, p$ are convex and continuously differentiable functions. The vectors $l, u \in \mathbb{R}^{n}$ are lower and upper bounds on the decision variables $x \in \mathbb{R}^{n}$ and define the box $B$. The index set $I \subseteq\{1, \ldots, n\}$ specifies which variables have to take integer values. We assume w.l.o.g. $l_{i}, u_{i} \in \mathbb{Z}$ for all $i \in I$. Note that problem (MOMIC) is nonconvex because of the presence of integrality constraints. However, when all the integer variables are fixed it reduces to a continuous convex problem as both the objective and constraint functions are convex. This is the reason why we call Problem (MOMIC) a multiobjective mixed integer convex programming problem. The image of the feasible set of the problem under the vector-valued function $f: \mathbb{R}^{n} \rightarrow \mathbb{R}^{m}$ represents the feasible set in the criterion space, or the image set.

Multiobjective mixed integer optimization problems arise in many application fields such as location or production planning, finance, manufacturing, and emergency management (see e.g. [18, 32, 39]). As an example we can think of the uncapacitated facility location problem, studied in the single-objective case in [23]. The first objective hereby is to decide which facilities to build in order to minimize costs. As a second objective function one could consider the total negative impact on the environment with the building plan for the facilities, e.g. the carbon emissions.

Solving a multiobjective optimization problem aims at detecting the efficient set, namely the set of points in the decision space that leads to nondominated points in the criterion space. A point of the image set is nondominated if none of its components can be decreased without increasing any other component. A formal definition will be given in Section 2.

It is well known that mixed integer nonlinear optimization is NP-hard and its solution typically requires dealing with enormous search trees [2]. Handling more than one objective function adds an additional difficulty: assume there is only one binary variable, $I=\{1\}$ with $x_{1} \in\{0,1\}$, and we have just one objective function, i. e., $m=1$. Then for solving (MOMIC) only two convex optimization problems have to be addressed, one with $x_{1}$ fixed to 0 and one with $x_{1}$ fixed to 1 . Clearly, the smallest minimal value is the optimal value of the original problem. In case of two or more objective functions already this simple setting is much more challenging. Solving the problems with fixed values for $x_{1}$ would mean to determine the whole efficient set of a multiobjective convex optimization problem, which is in general infinite. Then, after computing two sets of nondominated points one has to compare them and to determine the "smallest" values, see Figure 1 on page 5 for an illustration of this observation for four choices of the integer variables.

So far, most of the existing algorithms for multiobjective mixed integer optimization are only for linear programming problems. Those can be divided into two main classes: decision space search algorithms, i.e., approaches that work in the space of feasible points, and criterion space search algorithms, i.e., methods that work in the space of objective function values.

Among the decision space search algorithms, the method proposed by Mavrotas and Diakoulaki, [28], is the first branch-and-bound algorithm for solving multiobjective mixed binary programs. The authors improved and extended their work in [29]. Other works defining branch-and-bound algorithms for multiobjective integer linear programming problems are $[16,37]$. Branch-and-bound algorithms for biobjective mixed integer linear optimization problems are proposed in $[1,36]$. 
Criterion space search algorithms find nondominated points by addressing a sequence of single-objective optimization problems. Once a nondominated point is computed, dominated parts of the criterion space are removed and the algorithms continue the search for new nondominated points. Several contributions in the context of criterion space search algorithms for biobjective and triobjective integer linear programming are given by Boland and co-authors [3, 4, 5, 6]. For criterion space search algorithms for biobjective mixed integer linear problems see, for instance, [8, 9, 33, 38], for biobjective mixed binary linear problems, see [19], and for three objective functions, see [34].

As far as we know, the first general purpose method to tackle multiobjective mixed integer convex programs is the heuristic approach based on a branch-and-bound algorithm proposed by Cacchiani and D'Ambrosio in [14].

A classical technique to solve a multiobjective optimization problem is to convert the problem into a parameter-dependent single-objective one, known as scalarization. The scalarized problems are then parameter-dependent single-objective mixed integer convex optimization problems. By following this approach, many of these single-objective problems have to be solved, one for each choice of the parameter's value. It is a challenge to choose the parameter's values in a smart way, especially for $m \geq 3$, as the set of nondominated points is in general disconnected and can have huge gaps. Burachik et al. [11] recently proposed an algorithm that considers scalarized problems for multiobjective integer and mixed-integer optimization. It is based on the use of a clever grid generation technique for the parameters based on [12, 13]. Moreover, to speed up the algorithm, the optimal solution obtained at a neighboring grid point can be used as a good starting point. However, any other information regarding the branching and discarding within one run of the single-objective mixed integer sub-solver is not re-used. Thus, valuable information about promising subboxes, either for discarding or for good upper bounds, is lost.

We propose in this paper for the first time a non-scalarization based deterministic algorithm for multiobjective mixed integer convex problems which is not using a scalarization of the original problem. We directly develop a branch-and-bound algorithm based on a partitioning of the feasible region, i.e., a decision space algorithm. We present two versions of the algorithm. In one version we do not have to solve any single-objective mixed integer subproblem but only single-objective convex subproblems. In the second version we need to address also single-objective mixed integer convex optimization problems. No parameter needs to be chosen to define the subproblems we consider.

To compute lower bounds in our branch-and-bound approach, we rely on linear outer approximations of the image set. We use outer approximation techniques from convex multiobjective optimization for finding lower bounds of the continuous relaxation of the problem (i. e., the problem obtained by ignoring the integrality constraints), as well as for constructing outer approximations of the convex hull of the true image set over subboxes. First promising tests on bounds by continuous relaxations are reported in the master thesis of the fourth author [35]. We keep track of upper bounds in the image space and derive by that a discarding test for the branch-and-bound procedure. This results in a deterministic solver for which we can give theoretical guarantees to find approximations of the set of efficient and of the set of nondominated points. The algorithm is related to the one proposed in the context of multiobjective continuous nonconvex optimization [30] by the second and the third authors of this paper. Both algorithms are in fact branch-and- 
bound methods and are based on similar criteria to explore the feasible region and prune the nodes in the branching tree. The difference is that now the relaxations are obtained by ignoring the integrality assumptions - and that the bounds obtained thereby are improved by considering the convex hull of the true image sets.

The paper is organized as follows. In Section 2 we report notations and definitions that will be used throughout the paper. In Section 3 we present our branch-and-bound algorithm MOMIX. Details on how to define a "light" version of the algorithm that does not need to address any single-objective mixed integer convex programming problem are given as well. Theoretical insights of MOMIX and MOMIX $x_{\text {light }}$ are also given in Section 3. Some numerical results are reported in Section 4. Section 5 concludes the paper.

\section{Definitions and Notations}

Throughout the paper, we indicate with $\|\cdot\|$ the Euclidean norm. Given a box $B=[l, u]$, we denote by $\omega(B)$ its width obtained as the Euclidean distance between $l$ and $u$, namely $\omega(B)=\|u-l\|$. Given two vectors $x, y \in \mathbb{R}^{n}$, we write $x \leq y$ and $x<y$ if $x_{i} \leq y_{i}$ and $x_{i}<y_{i}$ for all $i \in\{1, \ldots, n\}$, respectively. We write $x \not \leq y$ when an index $i \in\{1, \ldots, n\}$ exists such that $x_{i}>y_{i}$. Given a vector $x \in \mathbb{R}^{n}$ and an index set $I \subseteq\{1, \ldots, n\}$, we denote with $x_{I}$ the subvector with components $x_{i}, i \in I$.

Let $x \in \mathbb{R}$, we define

$$
\begin{aligned}
& \lfloor x\rfloor:=\max \{c \in \mathbb{Z} \mid c \leq x\} \\
& \lceil x\rceil:=\min \{c \in \mathbb{Z} \mid x \leq c\}
\end{aligned} \quad \text { and } \quad[x]:= \begin{cases}\lceil x\rceil & \text { if } x+0.5 \geq\lceil x\rceil \\
\lfloor x\rfloor & \text { otherwise. }\end{cases}
$$

For $x \in \mathbb{R}^{n}$, we define $\lfloor x\rfloor,\lceil x\rceil$ and $[x]$ componentwise.

For a nonempty set $A \subset \mathbb{R}^{m}$, we denote by $\operatorname{conv}(A)$ the convex hull of $A$, namely the smallest convex set that contains $A$. The set int $(A) \operatorname{describes}$ the interior of the set $A$. By $B^{g}, B^{\mathbb{Z}}$ and $B^{g, \mathbb{Z}}$ we denote the following sets related to the constraints in (MOMIC):

$$
\begin{aligned}
& B^{g}:=\{x \in B \mid g(x) \leq 0\}, \\
& B^{\mathbb{Z}}:=\left\{x \in B \mid x_{i} \in \mathbb{Z} \text { for all } i \in I\right\}, \\
& B^{g, \mathbb{Z}}:=B^{g} \cap B^{\mathbb{Z}} .
\end{aligned}
$$

Using these sets, we can write (MOMIC) in short form as

$$
\begin{aligned}
& \min f(x) \\
& \text { s.t. } x \in B^{g, \mathbb{Z}} .
\end{aligned}
$$

As mentioned, we are going to define a branch-and-bound method based on partitioning the feasible set of (MOMIC). Our branching rule is based on bisections of the box $B$. Let $\tilde{B}$ be a subbox of $B$. By $\tilde{B}^{g}, \tilde{B}^{\mathbb{Z}}$ and $\tilde{B}^{g, \mathbb{Z}}$ we denote the sets defined according to (1), where the set $B$ is replaced by $\tilde{B}$ (i.e., $x \in \tilde{B}$ in all the set definitions).

We recall here the basic concepts of efficient and nondominated points (see [25] for further details).

\section{Definition 2.1}




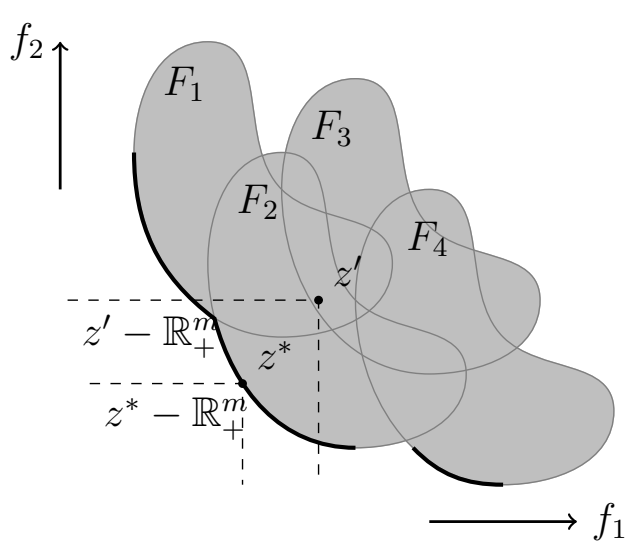

Figure 1: Image set of a biobjective instance of (MOMIC).

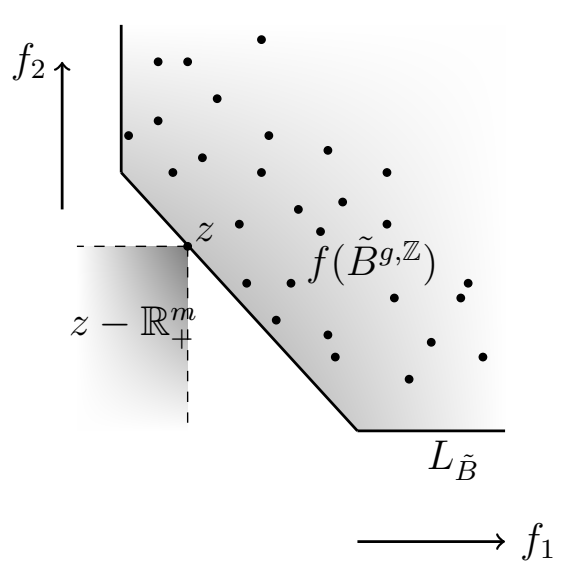

Figure 2: Image set of a biobjective purely integer instance of (MOMIC).

(a) A feasible point $x^{*} \in B^{g, \mathbb{Z}}$ is efficient for (MOMIC) if there is no $x \in B^{g, \mathbb{Z}}$ with $f(x) \leq f\left(x^{*}\right)$ and $f(x) \neq f\left(x^{*}\right)$. The set of efficient points for (MOMIC) is the efficient set of (MOMIC).

(b) A point $z^{*}=f\left(x^{*}\right)$ is nondominated for (MOMIC) if $x^{*} \in B^{g, \mathbb{Z}}$ is an efficient point for (MOMIC). The set of all nondominated points of (MOMIC) is the nondominated set of (MOMIC).

(c) Let $x, x^{*} \in B^{g, \mathbb{Z}}$ with $f(x) \leq f\left(x^{*}\right)$ and $f(x) \neq f\left(x^{*}\right)$. Then we say that $x$ dominates $x^{*}$ and also that $f(x)$ dominates $f\left(x^{*}\right)$.

In Figure 1, we plot the image set of a biobjective mixed integer convex optimization problem. Here, we assume that $\left\{x_{I} \mid x \in B^{g, \mathbb{Z}}\right\}=:\left\{y^{1}, y^{2}, y^{3}, y^{4}\right\}$ and we show the sets $F_{j}:=\left\{f(x) \mid x \in B^{g, \mathbb{Z}}, x_{I}=y^{j}\right\}, j=1, \ldots, 4$. Then the union of all $F_{j}$ describes the whole image set, i.e., $\bigcup_{j=1, \ldots, 4} F_{j}=\left\{f(x) \mid x \in B^{g, \mathbb{Z}}\right\}$. The point $z^{*} \in f\left(B^{g, \mathbb{Z}}\right)$ is nondominated and the preimage of $z^{*}$ is an efficient point. On the other hand, $z^{\prime} \in f\left(B^{g, \mathbb{Z}}\right)$ is dominated because $z^{*} \leq z^{\prime}$ and $z^{*} \neq z^{\prime}$. In fact, all the points $z \in F_{3}$ are dominated, as points $w \in f\left(B^{g, \mathbb{Z}}\right)$ exist such that $w<z$. The nondominated set of the problem is visualized as the thick boundary of the image set. The efficient set is made of all preimages of the nondominated set. Figure 1 shows that the nondominated set of a multiobjective mixed integer nonlinear programming problem is in general a disconnected set. From an algorithmic point of view, this makes the detection of the efficient set of (MOMIC) an extremely challenging problem. Furthermore, there is the necessity of comparing sets of points: this is a crucial difference with respect to single-objective mixed integer nonlinear optimization. 


\section{MOMIX: An Outer Approximation based Branch-and- Bound Algorithm for (MOMIC)}

The algorithm we propose is a branch-and-bound method that looks for the efficient set of (MOMIC) by partitioning the box $B$. At every node of the branch-and-bound tree, a subbox $\tilde{B} \subseteq B$ is selected and lower and upper bounds on the nondominated set of (MOMIC) are derived. When considering the subbox $\tilde{B}$, a lower bound is any set $L_{\tilde{B}} \subseteq \mathbb{R}^{m}$ such that $L_{\tilde{B}}+\mathbb{R}_{+}^{m}$ contains the image of feasible points $\tilde{B}^{g, \mathbb{Z}}$ through $f$, namely $f\left(\tilde{B}^{g, \mathbb{Z}}\right) \subseteq L_{\tilde{B}}+\mathbb{R}_{+}^{m}$. In Figure 2, we illustrate the set $f\left(\tilde{B}^{g, \mathbb{Z}}\right)$ and a lower bound $L_{\tilde{B}}$ for a biobjective purely integer programming problem: note that the image of feasible points in $\tilde{B}$ through $f$ is a set of isolated points in $\mathbb{R}^{m}$.

In our algorithm we derive lower bounds by building linear outer approximations of $\operatorname{conv}\left(f\left(\tilde{B}^{g, \mathbb{Z}}\right)\right)$. As $f\left(\tilde{B}^{g, \mathbb{Z}}\right) \subseteq \operatorname{conv}\left(f\left(\tilde{B}^{g, \mathbb{Z}}\right)\right)$ holds, linear outer approximations of the convex hull of $f\left(\tilde{B}^{g, \mathbb{Z}}\right)$ are valid lower bounds on $\tilde{B}$. Details on how we compute the hyperplanes to outer approximate $\operatorname{conv}\left(f\left(\tilde{B}^{g, \mathbb{Z}}\right)\right)$ will be given in Section 3.2.

Upper bounds are computed by evaluating the objective functions at feasible points. As soon as an upper bound $z$ exists such that $L_{\tilde{B}}+\mathbb{R}_{+}^{m} \subseteq\{z\}+\mathbb{R}_{+}^{m} \backslash\{0\}$ we can discard the subbox $\tilde{B}$. Or, in other words, we can avoid to go on partitioning $\tilde{B}$, as we have an evidence that it cannot contain any efficient point for (MOMIC). Our discarding procedure is in fact using a list of upper bounds and it will be detailed in Section 3.1 and Section 3.2.

In Figure 2, the point $z \in f\left(\tilde{B}^{g, \mathbb{Z}}\right)$ is an upper bound for the nondominated set of the problem, as it is the image of a feasible point. All the points that belong to $\mathbb{R}^{m} \backslash(\{z\}+$ $\left.\mathbb{R}_{+}^{m} \backslash\{0\}\right)$ are candidates to belong to the nondominated set (note that it is not enough to consider $\left.\{z\}-\mathbb{R}_{+}^{m}\right)$.

Let $\delta>0$ be a positive scalar, which is the input parameter of our branch-and-bound method. As the output of our algorithm, we will have a list of subboxes $\tilde{B}$ with $\omega(\tilde{B})<\delta$, containing the set of all efficient points, and a list of upper bounds approximating the nondominated set.

Algorithm 1 is a basic scheme of our branch-and-bound procedure: $\mathcal{L}_{\mathcal{W}}$ denotes the working list and contains boxes that still have to be examined. The list $\mathcal{L}_{\mathcal{S}}$ denotes the list of boxes that fulfill the termination criteria, i.e., those subboxes $\tilde{B}$ that were not discarded and satisfy $\omega(\tilde{B})<\delta$. In Section 3.3 we will prove that $\mathcal{L}_{\mathcal{S}}$ represents a cover of

the efficient set $E$, namely $E \subseteq \bigcup_{\tilde{B} \in \mathcal{L}_{\mathcal{S}}} \tilde{B}$. The list $\mathcal{L}_{P N S}$ denotes a set of upper bounds and it will be defined in Section 3.1. Note that the flag $\mathcal{D}$ is used in order to decide if a box should be discarded, and it is an output of Algorithm 2. As a final step in Algorithm 1 we filter the list $\mathcal{L}_{\mathcal{S}}$ by a postprocessing phase. Further details will be given in Section 3.2.

\subsection{Computation of upper bounds and local upper bounds}

In order to compute upper bounds of the nondominated set of (MOMIC), we evaluate the objective functions at feasible points $x \in B^{g, \mathbb{Z}}$. It is well known that determining feasible points of a mixed integer set is an NP-hard problem. In the literature, several heuristic methods have been proposed. The most popular one is the Feasibility Pump [20] and some of its enhancements, among them [7, 10, 15, 21]. Within our algorithm, we either detect 


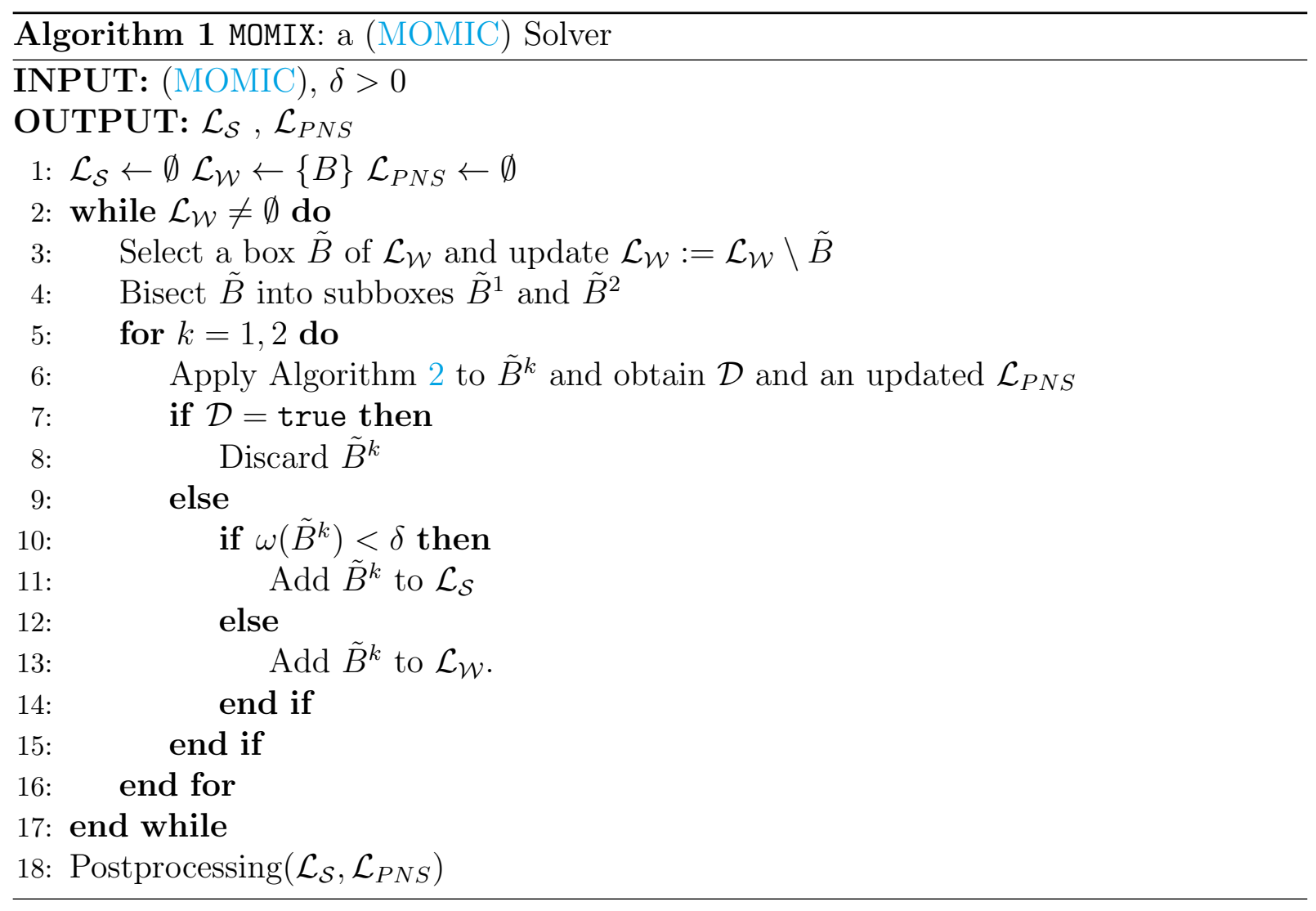

feasible points by addressing specific single-objective mixed integer convex programming problems (see Section 3.2) or we try to build feasible points simply by rounding the integer components of points $x \in \tilde{B}$, which are generated in our discarding test. Let round $(x)$ be the point defined as

$$
(\operatorname{round}(x))_{i}=\left\{\begin{array}{ll}
{\left[x_{i}\right]} & i \in I \\
x_{i} & \text { otherwise, }
\end{array} \quad \text { for each } i \in\{1, \ldots, n\}\right.
$$

If $\operatorname{round}(x) \in B^{g, \mathbb{Z}}$ holds, $f(\operatorname{round}(x))$ is a valid upper bound.

Upper bounds are needed in order to discard boxes or, in other words, to prune nodes in the branch-and-bound tree. In order to do that we need to introduce two finite sets of points, namely the list of potentially nondominated solutions $\mathcal{L}_{P N S} \subseteq f\left(B^{g, \mathbb{Z}}\right)$ and the list of local upper bounds $\mathcal{L}_{L U B} \subseteq \mathbb{R}^{m}$.

In our algorithm the list of potentially nondominated solutions $\mathcal{L}_{P N S}$ is initialized as the empty set. Then, every time an upper bound $z \in f\left(B^{g, \mathbb{Z}}\right)$ is computed, we check whether it is dominated by any point in $\mathcal{L}_{P N S}$. If this is the case, $z$ is not added to $\mathcal{L}_{P N S}$. Otherwise, we update the list by adding $z$ to $\mathcal{L}_{P N S}$ and by removing all the upper bounds dominated by $z$ from $\mathcal{L}_{P N S}$. By doing this, we ensure that $\mathcal{L}_{P N S}$ is a stable set of points: a set $\mathcal{N} \subseteq \mathbb{R}^{m}$ is said to be stable if there are no $x, y \in \mathcal{N}$ with $x \leq y$ and $x \neq y$.

For the list of local upper bounds $\mathcal{L}_{L U B}$ we need the following definition:

Definition 3.1 [26] Let $\mathcal{N} \subseteq f(B)$ be a finite and stable set of points and $Z \subseteq \mathbb{R}^{m}$ be a box such that $f(B) \subseteq \operatorname{int}(Z)$. 
(a) The search region related to $\mathcal{N}$ and $Z$ is defined as

$$
S:=\{w \in \operatorname{int}(Z) \mid z \not \leq w \text { for all } z \in \mathcal{N}\} .
$$

(b) The search zone for some $p \in \mathbb{R}^{m}$ related to $Z$ is defined as

$$
C(p)=\{w \in \operatorname{int}(Z) \mid w<p\} .
$$

(c) A list $\mathcal{L} \subseteq Z$ is called a local upper bound set with respect to $\mathcal{N}$, if

(i) $S=\bigcup_{p \in \mathcal{L}} C(p)$

(ii) $C(p)$ is not a subset of $C(\tilde{p})$ for all $p, \tilde{p} \in \mathcal{L}$.

Let $Z \subseteq \mathbb{R}^{m}$ be a box such that $f(B) \subseteq \operatorname{int}(Z)$. In our algorithm we initialize the local upper bound set $\mathcal{L}_{L U B}$ with the point $p^{0} \in \mathbb{R}^{m}$ defined as $p_{j}^{0}:=\max _{w \in Z} w_{j}$. Then we build and keep updated $\mathcal{L}_{L U B}$ with respect to the finite and stable set $\mathcal{L}_{P N S}$ according to the procedure proposed in [26]. For an illustration of the local upper bound set $\mathcal{L}_{L U B}$ with respect to $\mathcal{L}_{P N S}$, see Figure 3 on page 9 .

Remark 3.2 Along the iterations of our algorithm, let $\mathcal{L}_{P N S}^{\prime}$ and $\mathcal{L}_{P N S}$ be two consecutive lists of potentially nondominated points, i. e., $\mathcal{L}_{P N S}^{\prime}$ is the set of potentially nondominated solutions during an earlier iteration than $\mathcal{L}_{P N S}$, which evolved from $\mathcal{L}_{P N S}^{\prime}$ along the algorithm. Let $\mathcal{L}_{L U B}^{\prime}$ and $\mathcal{L}_{L U B}$ be the related local upper bound sets. Then, based on the update procedure mentioned above, we have that to any (former) $z^{\prime} \in \mathcal{L}_{P N S}^{\prime}$ there exists (a current) $z \in \mathcal{L}_{P N S}$ with $z \leq z^{\prime}$. Hence, the search regions $S^{\prime}$ and $S$ related to $\mathcal{L}_{P N S}^{\prime}$ and $\mathcal{L}_{P N S}$ are such that $S \subseteq S^{\prime}$, i.e.,

$$
S=\bigcup_{p \in \mathcal{L}_{L U B}} C(p) \subseteq \bigcup_{p \in \mathcal{L}_{L U B}^{\prime}} C(p)=S^{\prime} .
$$

Furthermore, for every (current) $p \in \mathcal{L}_{L U B}$ there exists a (former) local upper bound $p^{\prime} \in \mathcal{L}_{L U B}^{\prime}$ such that $p \leq p^{\prime}$. This can be seen by induction considering the updating procedure proposed in [26].

Note that $p \in \mathcal{L}_{L U B}$ is not necessarily the image of a feasible point. Local upper bounds are used in order to decide if a subbox $\tilde{B} \subseteq B$ should be discarded as clarified by the following results.

Lemma 3.3 [30] Let $\mathcal{L}_{L U B}$ be a local upper bound set with respect to the finite and stable set $\mathcal{L}_{P N S} \subseteq f\left(B^{g, \mathbb{Z}}\right)$. For every $z \in \mathcal{L}_{P N S}$ and for every $j \in\{1, \ldots, m\}$ there is a $p \in \mathcal{L}_{L U B}$ with $z_{j}=p_{j}$ and $z_{r}<p_{r}$ for all $r \in\{1, \ldots, m\} \backslash\{j\}$.

Based on this lemma we can prove our main result for the pruning of nodes:

Theorem 3.4 Consider a subbox $\tilde{B} \subseteq B$. Let $\mathcal{L}_{P N S} \subseteq f\left(B^{g, \mathbb{Z}}\right)$ be a finite and stable set. Let $\mathcal{L}_{L U B}$ be the local upper bound set w.r.t. $\mathcal{L}_{P N S}$. If

$$
p \notin f\left(\tilde{B}^{g, \mathbb{Z}}\right)+\mathbb{R}_{+}^{m} \quad \text { holds for all } p \in \mathcal{L}_{L U B},
$$

$\tilde{B}$ does not contain any efficient point for (MOMIC). 


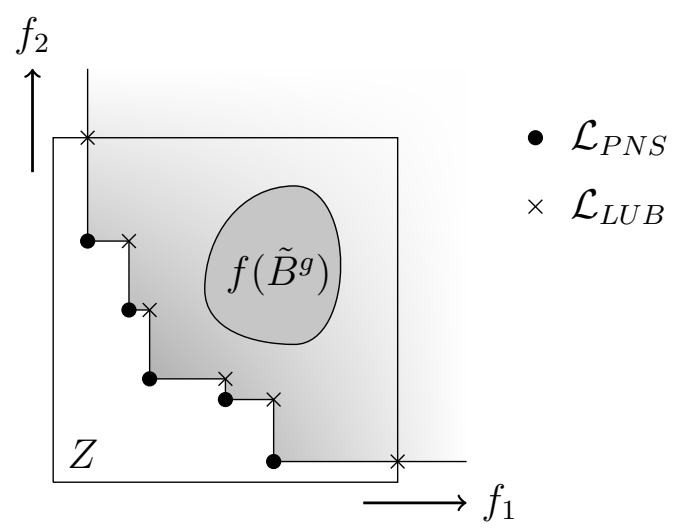

Figure 3: Illustration of Corollary 3.5 for $m=2$. In the picture we plot the local upper bound set $\mathcal{L}_{L U B}$ with respect to the set of potentially nondominated solutions $\mathcal{L}_{P N S}$. Note that the box $\tilde{B}$ would be discarded, as the assumptions of Corollary 3.5 (a) are satisfied and $\tilde{B}$ cannot contain any efficient point for (MOMIC).

Proof. Assume by contradiction that an efficient point $x^{*} \in \tilde{B}^{g, \mathbb{Z}}$ for (MOMIC) exists. From (2), we have

$$
f\left(x^{*}\right) \not \leq p \text { for all } p \in \mathcal{L}_{L U B} .
$$

Since $\mathcal{L}_{L U B}$ is a local upper bound set w.r.t. $\mathcal{L}_{P N S}$, it follows from (3) and Definition 3.1 (b) and (c), that $f\left(x^{*}\right)$ does not belong to the search region $S$. Hence, there exists a point $z \in \mathcal{L}_{P N S}$ with $z \leq f\left(x^{*}\right)$. As $z \in \mathcal{L}_{P N S}$, a point $x^{\prime} \in B^{g, \mathbb{Z}}$ exists such that $z=f\left(x^{\prime}\right)$. Since $x^{*}$ is efficient for (MOMIC), it follows $z=f\left(x^{\prime}\right)=f\left(x^{*}\right)$. Lemma 3.3 implies that there is a point $p^{\prime} \in \mathcal{L}_{L U B}$ with $f\left(x^{*}\right) \leq p^{\prime}$, which is a contradiction to (3) and the theorem is proved.

From Theorem 3.4, since $\tilde{B}^{g, \mathbb{Z}} \subseteq \tilde{B}^{g}$ and $f\left(\tilde{B}^{g, \mathbb{Z}}\right) \subseteq \operatorname{conv}\left(f\left(\tilde{B}^{g, \mathbb{Z}}\right)\right)$ hold, we obtain the following corollary.

Corollary 3.5 Let $\tilde{B}$ be a subbox of $B$. Let $\mathcal{L}_{P N S} \subseteq f\left(B^{g, \mathbb{Z}}\right)$ be a finite and stable set and let $\mathcal{L}_{L U B}$ be the local upper bound set w.r.t. $\mathcal{L}_{P N S}$.

(a) If

$$
p \notin f\left(\tilde{B}^{g}\right)+\mathbb{R}_{+}^{m} \quad \text { holds for all } p \in \mathcal{L}_{L U B},
$$

$\tilde{B}$ does not contain any efficient point for (MOMIC).

(b) If

$$
p \notin \operatorname{conv}\left(f\left(\tilde{B}^{g, \mathbb{Z}}\right)\right)+\mathbb{R}_{+}^{m} \quad \text { holds for all } p \in \mathcal{L}_{L U B},
$$

$\tilde{B}$ does not contain any efficient point for (MOMIC).

An illustration of Corollary 3.5 (a) can be found in Figure 3.

The following remark clarifies how the assumptions of Corollary 3.5 are related. Furthermore, it gives the basis of the hierarchy of lower bounds in our bounding procedure.

Remark 3.6 Note that due to the convexity of the objective functions $f_{j}, j=1, \ldots, m$ and of $\tilde{B}^{g}$ the following holds

$$
\operatorname{conv}\left(f\left(\tilde{B}^{g, \mathbb{Z}}\right)\right)+\mathbb{R}_{+}^{m} \subseteq f\left(\tilde{B}^{g}\right)+\mathbb{R}_{+}^{m}
$$




\subsection{Determining lower bounds and pruning nodes}

The theoretical results introduced in the previous section, namely Corollary 3.5, give the basis of the discarding procedure in our branch-and-bound algorithm: For every subbox $\tilde{B}$ we want to check whether $p \notin L_{\tilde{B}}+\mathbb{R}_{+}^{m}$ holds for all $p \in \mathcal{L}_{L U B}$, being $L_{\tilde{B}}$ a valid lower bound for $\tilde{B}^{g, \mathbb{Z}}$.

As $f\left(\tilde{B}^{g}\right)$ is a valid lower bound, a straightforward way to verify if a box should be discarded would be to check whether a local upper bound $p \in \mathcal{L}_{L U B}$ belongs to this lower bound given by the convex relaxation. This would mean to check whether $p \in$ $f\left(\tilde{B}^{g}\right)+\mathbb{R}_{+}^{m}$ holds. This can be done by addressing a simple single-objective continuous convex problem.

From a computational point of view, this means that we would need to solve $\left|\mathcal{L}_{L U B}\right|$ single-objective continuous convex problems, at every node of the branching tree.

In our algorithm, in order to reduce this numerical effort, we check instead whether a local upper bound belongs to a linear outer approximation of $f\left(\tilde{B}^{g}\right)+\mathbb{R}_{+}^{m}$, i.e., we only need to check whether a local upper bound satisfies linear inequalities. Furthermore, our linear outer approximations are built in a smart way: the supporting hyperplanes computation is adaptively driven by some "meaningful" local upper bounds $p \in \mathcal{L}_{L U B}$.

Additionally, if we want to improve our lower bound, we compute further hyperplanes to outer approximate $\operatorname{conv}\left(f\left(\tilde{B}^{g, \mathbb{Z}}\right)\right)+\mathbb{R}_{+}^{m}$. Again this computation is done in an adaptive way, and the supporting hyperplanes computation is steered by some specific local upper bounds $p \in \mathcal{L}_{L U B}$.

In the following, we give details on how the supporting hyperplanes are computed and how the discarding procedure works.

At an arbitrary node of our branching tree we select a subbox $\tilde{B} \subseteq B$. In order to compute valid lower bounds on $\tilde{B}$ we build linear outer approximations $L_{\tilde{B}}$ of $\operatorname{conv}\left(f\left(\tilde{B}^{g, \mathbb{Z}}\right)\right)$, such that

$$
f\left(\tilde{B}^{g, \mathbb{Z}}\right) \subseteq \operatorname{conv}\left(f\left(\tilde{B}^{g, \mathbb{Z}}\right)\right) \subseteq L_{\tilde{B}}+\mathbb{R}_{+}^{m} .
$$

In order to discard the subbox $\tilde{B}$ and prune the current node, we check whether

$$
p \notin L_{\tilde{B}}+\mathbb{R}^{m} \quad \text { holds for all } p \in \mathcal{L}_{L U B} \text {. }
$$

Then, from Corollary 3.5 $\tilde{B}$ does not contain any efficient point for (MOMIC) and the current node can be pruned. As we will deal with linear outer approximations of sets, recall here the definition of supporting hyperplane of a set:

Definition 3.7 Let $P \subset \mathbb{R}^{m}$ be a nonempty set, let $\lambda \in \mathbb{R}^{m} \backslash\{0\}$ and $z \in \partial P$, where $\partial P$ is the boundary of the set $P$. The hyperplane

$$
H^{\lambda, z}:=\left\{y \in \mathbb{R}^{m} \mid \lambda^{T} y=\lambda^{T} z\right\}
$$

is called supporting hyperplane (of $P$ ), if $\lambda^{T} y \geq \lambda^{T} z$ holds for all $y \in P$.

As mentioned in the introduction, we propose two versions of our branch-and-bound algorithm. The difference lies in the lower bounds computation. The first version of our algorithm, named MOMIX $\mathrm{light}_{\text {, }}$, computes valid lower bounds by addressing only singleobjective continuous convex optimization problems. The second version, named MOMIX, 
tries to define stronger lower bounds by dealing also with single-objective mixed integer convex programming problems. In our algorithm we use a flag light to distinguish between the two versions of the method.

Both, MOMIX $\mathrm{I}_{\text {light }}$ and MOMIX, start by computing linear outer approximations of the convex set $f\left(\tilde{B}^{g}\right)+\mathbb{R}_{+}^{m}$ by solving a family of single-objective continuous convex optimization problems. As $\operatorname{conv}\left(f\left(\tilde{B}^{g, \mathbb{Z}}\right)\right)+\mathbb{R}_{+}^{m} \subseteq f\left(\tilde{B}^{g}\right)+\mathbb{R}_{+}^{m}$ holds by Remark 3.6, we have that linear outer approximations of $f\left(\tilde{B}^{g}\right)+\mathbb{R}_{+}^{m}$ are valid lower bounds for $\operatorname{conv}\left(f\left(\tilde{B}^{g, \mathbb{Z}}\right)\right)$ as well (see Figure 4 on page 13). If the linear outer approximation of $f\left(\tilde{B}^{g}\right)+\mathbb{R}_{+}^{m}$ does not allow to discard the box $\tilde{B}$, MOMIX tries to improve it by addressing properly defined single-objective mixed integer convex programming problems.

As a first step for the outer approximation, we compute the ideal point $f^{i d} \in \mathbb{R}^{m}$ of $f\left(\tilde{B}^{g}\right)$, namely the point whose $j$-th component is the minimum of $f_{j}$ on $\tilde{B}^{g}$ :

$$
f_{j}^{i d}:=\min _{x \in \tilde{B}^{g}} f_{j}(x) \quad j=1, \ldots, m .
$$

We denote by $x^{j, i d} \in \tilde{B}^{g}$ a minimal solution in (4). Let $e^{j}$ be the $j$-th unit vector, then $H^{e^{j}, f^{i d}}$ is a supporting hyperplane of $f\left(\tilde{B}^{g}\right)$. As a first linear outer approximation of $f\left(\tilde{B}^{g}\right)$ or, in other words, as a first lower bound for $f\left(\tilde{B}^{g, \mathbb{Z}}\right)$ we consider

$$
L_{\tilde{B}}:=\partial\left(\bigcap_{j \in\{1, \ldots, m\}}\left(H^{e^{j}, f^{i d}}+\mathbb{R}_{+}^{m}\right)\right)=\left\{f^{i d}\right\}+\partial\left(\mathbb{R}_{+}^{m}\right) .
$$

Note that building $L_{\tilde{B}}$ requires to solve $m$ single-objective continuous convex optimization problems for the computation of $f^{i d}$.

Once $L_{\tilde{B}}$ is computed we enter in a loop. For every $p \in \mathcal{L}_{L U B}$ we check whether $p \in L_{\tilde{B}}+\mathbb{R}_{+}^{m}$ holds. If this is the case, we try to improve the current linear outer approximation $L_{\tilde{B}}$ by computing a further hyperplane, based on $p \in \mathcal{L}_{L U B}$. This is done by addressing the following single-objective continuous convex programming problem (see also $[17,27]$ )

$$
\begin{array}{ll}
\min & t \\
\text { s.t. } & f(x) \leq p+t e \\
& x \in \tilde{B}^{g} \\
& t \in \mathbb{R},
\end{array}
$$

where $e=(1, \ldots, 1)^{T} \in \mathbb{R}^{m}$.

Note that Problem $\left(\mathrm{P}_{p}\left(\tilde{B}^{g}\right)\right)$ needs to be addressed only in case of $p \in L_{\tilde{B}}+\mathbb{R}_{+}^{m}$. In other words, in our lower bound computation, we do not necessarily address Problem $\left(\mathrm{P}_{p}\left(\tilde{B}^{g}\right)\right)$ for all $p \in \mathcal{L}_{L U B}$, as it would be the case if we would rely only on the convex relaxation $f\left(\tilde{B}^{g}\right)$.

Under regularity assumptions, we have that any minimal solution $(\hat{x}, \hat{t}) \in \tilde{B}^{g} \times \mathbb{R}$ of Problem $\left(\mathrm{P}_{p}\left(\tilde{B}^{g}\right)\right)$ admits Lagrange multipliers. We refer to $[17,30]$ in case no Lagrange multiplier exists. Let $(\hat{x}, \hat{t}) \in \tilde{B}^{g} \times \mathbb{R}$ be a minimal solution of $\left(\mathrm{P}_{p}\left(\tilde{B}^{g}\right)\right)$ and let $\hat{\lambda} \in \mathbb{R}_{+}^{m}$ be a Lagrange multiplier for the constraint $f(x) \leq p+t e$. Then, the hyperplane $H^{\hat{\lambda}, \hat{y}(p)}$ with $\hat{y}(p):=p+\hat{t} e$ is a supporting hyperplane of $f\left(\tilde{B}^{g}\right)$, cf. [27, 31,30].

There exist two possibilities: 
(i) If $\hat{t}>0$ holds, then $p \notin f\left(\tilde{B}^{g}\right)+\mathbb{R}_{+}^{m}$, we improve the outer approximation by $H^{\hat{\lambda}, \hat{y}(p)}$, and consider the next local upper bound;

(ii) if $\hat{t} \leq 0$ holds, then $p \in f\left(\tilde{B}^{g}\right)+\mathbb{R}_{+}^{m}$ and the assumption of Corollary 3.5 (a) is not satisfied.

If case (ii) occurs, so far we cannot discard $\tilde{B}$ based on Corollary 3.5 (a) as it may contain efficient points for (MOMIC). Then, in case we apply MOMIX, i.e., light $=0$, we try to apply Corollary 3.5 (b) and thus we try to improve our linear outer approximation by addressing a single-objective mixed integer convex programming problem. Let $\hat{\lambda} \in \mathbb{R}^{m}$ be a Lagrange multiplier for the constraint $f(x) \leq p+t e$ for the solution of $\left(\mathrm{P}_{p}\left(\tilde{B}^{g}\right)\right)$. We define the following problem

$$
\begin{aligned}
& \min \hat{\lambda}^{T} f(x) \\
& \text { s.t. } x \in \tilde{B}^{g, \mathbb{Z}} .
\end{aligned}
$$

Let $\hat{x} \in \tilde{B}^{g, \mathbb{Z}}$ be a minimal solution of $\left(\operatorname{MICP}_{p}(\hat{\lambda}, \tilde{B})\right)$. Then the hyperplane $H^{\hat{\lambda}, f(\hat{x})}$ is a supporting hyperplane of $\operatorname{conv}\left(f\left(\tilde{B}^{g, \mathbb{Z}}\right)\right)$ and $\operatorname{conv}\left(f\left(\tilde{B}^{g, \mathbb{Z}}\right)\right)+\mathbb{R}_{+}^{m} \subseteq H^{\hat{\lambda}, f(\hat{x})}+\mathbb{R}_{+}^{m}$ holds. Furthermore, $f(\hat{x})$ is a valid upper bound for (MOMIC). Note that in case we are at a node where all integer variables are fixed we do not need to perform this step, because $\tilde{B}^{g \mathbb{Z}}=\tilde{B}^{g}$ holds.

Again two situations occur:

(i) If $\hat{\lambda}^{T} p<\hat{\lambda}^{T} f(\hat{x})$ holds, we improve the outer approximation by $H^{\hat{\lambda}, f(\hat{x})}$ and consider the next local upper bound

(ii) If $\hat{\lambda}^{T} p \geq \hat{\lambda}^{T} f(\hat{x})$ holds, the local upper bound $p$ lies above the hyperplane $H^{\hat{\lambda}, f(\hat{x})}$.

If we are in case (ii), we do not continue with improving the linear outer approximation and we branch the current node by bisecting $\tilde{B}$ in a later iteration.

Let $\mathcal{H}$ be the final linear outer approximation of $f\left(\tilde{B}^{g, \mathbb{Z}}\right)$ containing all computed supporting hyperplanes. Then the lower bound $L_{\tilde{B}}$ is represented by

$$
L_{\tilde{B}}:=\partial\left(\bigcap_{H \in \mathcal{H}}\left(H+\mathbb{R}_{+}^{m}\right)\right) .
$$

Algorithm 2 is reporting our lower bound computation in detail.

As soon as feasible points of $\tilde{B}^{g, \mathbb{Z}}$ are found, both $\mathcal{L}_{P N S}$ and $\mathcal{L}_{L U B}$ are updated. This is the reason why in Algorithm 2 we make use of the list $\mathcal{L}_{L U B}^{*}$ which does not change along the discarding test: We need a fixed set of local upper bounds in order to ensure the termination of the loop starting in line 10 .

Note that in line 17 we update the linear outer approximation even if the subbox $\tilde{B}$ is further kept either in the working list $\mathcal{L}_{\mathcal{W}}$ or in the solution list $\mathcal{L}_{\mathcal{S}}$. This is done in order to perform the postprocessing phase in Algorithm 1: Let $\tilde{B} \in \mathcal{L}_{\mathcal{S}}$ and let $\mathcal{H}$ be the linear outer approximation of $f\left(\tilde{B}^{g, \mathbb{Z}}\right)$ built by Algorithm 2. This subbox $\tilde{B}$ is removed from $\mathcal{L}_{\mathcal{S}}$ if for all local upper bounds $p$ belonging to the final list $\mathcal{L}_{L U B}$ we have that a hyperplane $H^{\lambda, z^{\prime}} \in \mathcal{H}$ exists such that $\lambda^{T} p \geq \lambda^{T} z^{\prime}$ holds. 


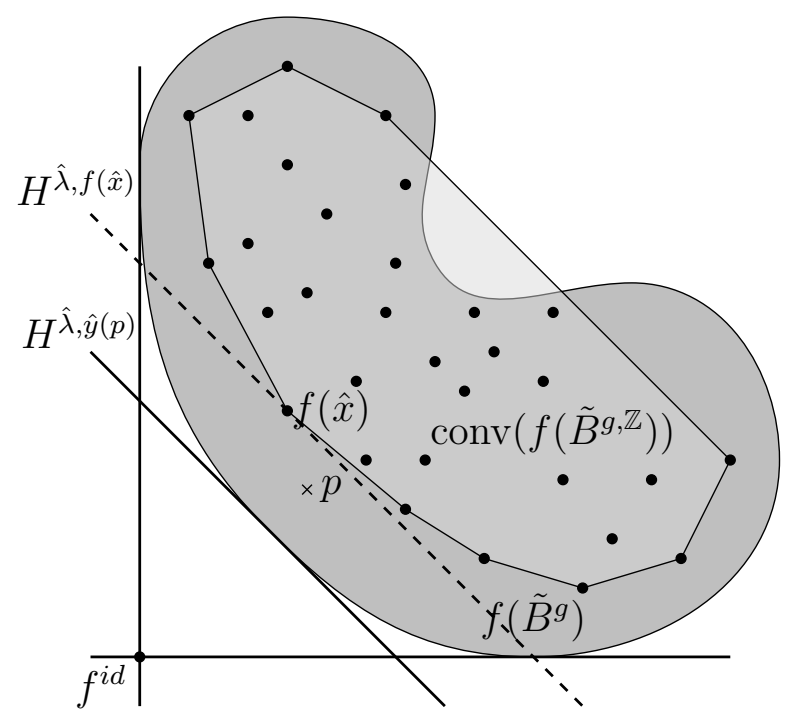

Figure 4: Illustration of our lower bounding procedure on a biobjective purely integer convex programming instance.

Example 3.8 In Figure 4 on page 13 we illustrate our lower bounding procedure on a biobjective purely integer convex programming instance. Note that in this case the image of feasible points is a set of isolated points in $\mathbb{R}^{2}$. The first outer approximation considered is based on the ideal point $f^{i d}$. Then, considering the local upper bound $p \in \mathcal{L}_{L U B}$, the supporting hyperplane $H^{\hat{\lambda}, \hat{y}(p)}$ for $f\left(\tilde{B}^{g}\right)$ is built by solving Problem $\left(\mathrm{P}_{p}\left(\tilde{B}^{g}\right)\right)$ and added to the linear outer approximation. Finally, in case MOMIX (and not MOMIX light) is applied, the linear outer approximation is further refined by considering $H^{\hat{\lambda}, f(\hat{x})}$, being $\hat{x}$ a solution of $\left(\operatorname{MICP}_{p}(\hat{\lambda}, \tilde{B})\right)$.

In the following lemma, we prove the exactness of our lower bounding procedure: we show that Algorithm 2 returns $\mathcal{D}=$ false in case $\tilde{B}$ contains an efficient point for (MOMIC). Thus it will be further partitioned.

Lemma 3.9 Let $\tilde{B}$ be a subbox of $B$ that contains an efficient point $x^{*} \in \tilde{B}^{g, \mathbb{Z}}$ of (MOMIC). Then Algorithm 2 returns $\mathcal{D}=$ false.

Proof. We distinguish two cases for which Algorithm 2 returns $\mathcal{D}=$ true: either $\mathcal{D}=$ true because $\left(\operatorname{MICP}_{p}(\hat{\lambda}, \tilde{B})\right)$ is infeasible for any $p \in \mathcal{L}_{L U B}^{*}$ or because lines 18 or 28 are never reached for any $p \in \mathcal{L}_{L U B}^{*}$. The first case cannot occur as $x^{*} \in \tilde{B}^{g, \mathbb{Z}}$. The second case may occur if for all $p \in \mathcal{L}_{L U B}^{*}$ either the condition in line 11 or the condition in line 16 or the condition in line 27 is not satisfied. In all three cases we get that $p \notin f\left(\tilde{B}^{g}\right)+\mathbb{R}_{+}^{m}$ holds for all $p \in \mathcal{L}_{L U B}^{*}$. Corollary 3.5 then implies that $\tilde{B}$ does not contain any efficient point for (MOMIC).

\subsection{Correctness of MOMIX}

We already mentioned that our algorithm stops as soon as the working list $\mathcal{L}_{\mathcal{W}}$ is empty and we get a list of subboxes $\tilde{B}$ of width less than a prescribed value $\delta>0$, i.e., $\omega(\tilde{B})<\delta$. 


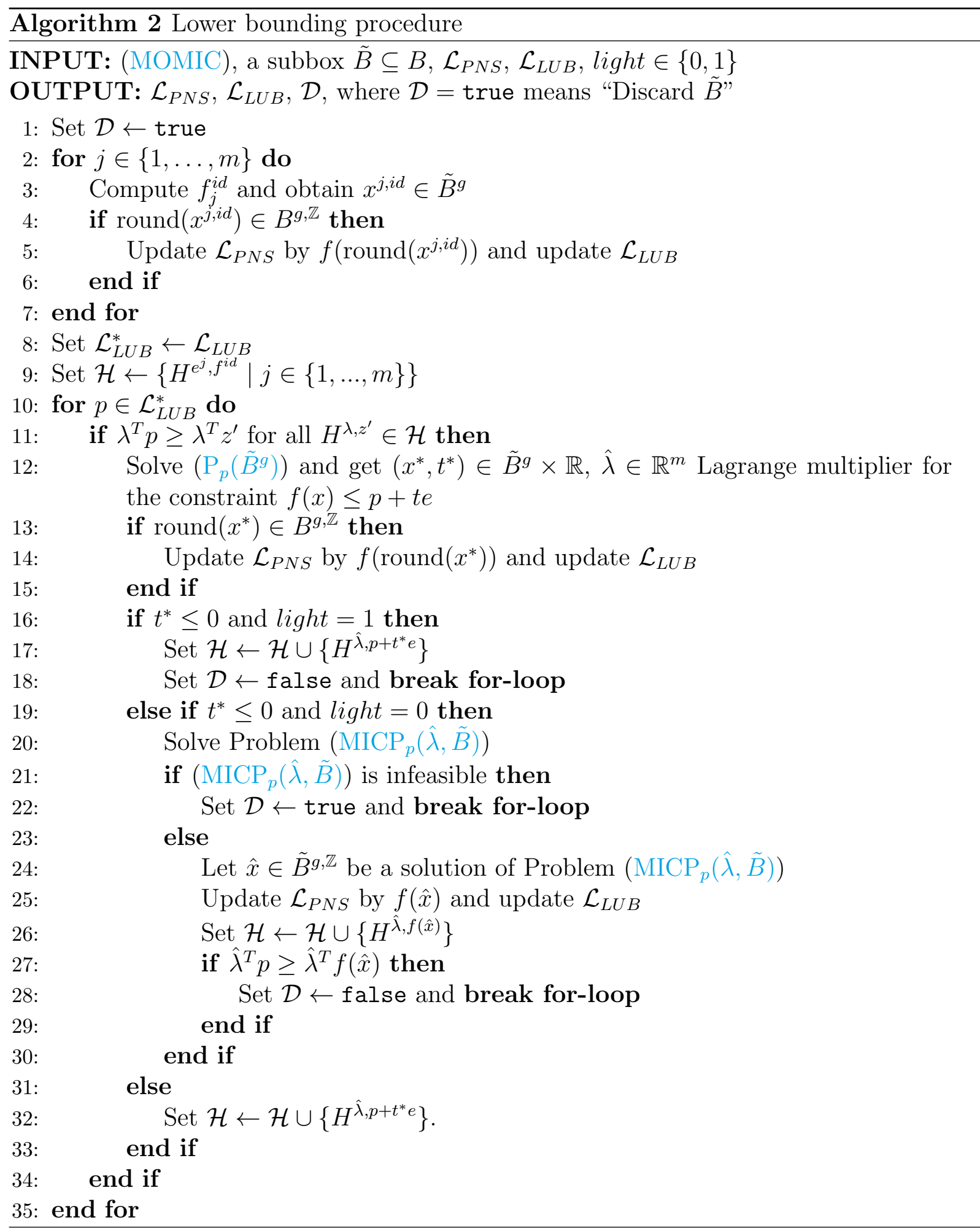


In this section, we show the exactness of Algorithm 1, namely we prove that it returns the set $\mathcal{L}_{\mathcal{S}}$ which is a cover of the efficient set $E$ of (MOMIC). In order to do that, we need to make the following assumption related to the branching rule adopted.

Assumption 3.10 Let $\tilde{B} \subseteq B$. Let the branching rule in Algorithm 1 be such that

$$
\tilde{B}^{g, \mathbb{Z}} \subseteq \tilde{B}^{1} \cup \tilde{B}^{2}
$$

holds for the subboxes $\tilde{B}^{1}$ and $\tilde{B}^{2}$ derived from $\tilde{B}$, and that the algorithm performs a finite number of branching steps before stopping.

Note that Assumption 3.10 implies that the set of efficient points for (MOMIC) belonging to $\tilde{B}$ is a subset of $\tilde{B}^{1} \cup \tilde{B}^{2}$. In Section 4 we propose and compare two branching rules which both satisfy Assumption 3.10.

From Assumption 3.10 and Lemma 3.9 we directly get the following

Theorem 3.11 Let $E$ be the efficient set of (MOMIC). Let $\mathcal{L}_{\mathcal{S}}$ be the output of Algorithm 1. Then $\mathcal{L}_{\mathcal{S}}$ is a cover of $E$, namely $E \subseteq \bigcup_{\tilde{B} \in \mathcal{L}_{\mathcal{S}}} \tilde{B}$.

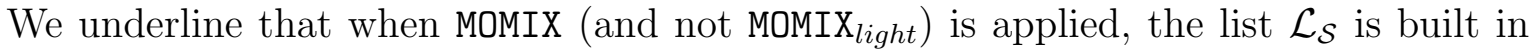
a way such that every subbox $\tilde{B}$ belonging to $\mathcal{L}_{\mathcal{S}}$ admits at least one feasible point, i.e., $\tilde{B}^{g, \mathbb{Z}} \neq \emptyset$. Note that a feasible point $\hat{x} \in \tilde{B}^{g, \mathbb{Z}}$ is computed in line 24 in Algorithm 2.

We further prove that the points in the final list of potentially nondominated solutions $\mathcal{L}_{P N S}$ are images of some points in the cover of the efficient set of (MOMIC).

Proposition 3.12 Let $\mathcal{L}_{P N S}$ and $\mathcal{L}_{\mathcal{S}}$ be the output of Algorithm 1. Then, for every $z \in \mathcal{L}_{P N S}$ there exists a subbox $\tilde{B} \in \mathcal{L}_{\mathcal{S}}$ such that $z \in f\left(\tilde{B}^{g, \mathbb{Z}}\right)$.

Proof. Assume by contradiction that the preimage $x$ of $z \in \mathcal{L}_{P N S}$ belongs to a discarded subbox $\tilde{B}$. We recall that the subbox $\tilde{B}$ is discarded if no local upper bound belongs to $L_{\tilde{B}}+\mathbb{R}_{+}^{m}$, as this implies that $\tilde{B}$ does not contain any efficient point for (MOMIC) (see Corollary 3.5).

This means that at a certain node of our branching tree, a lower bound $L_{\tilde{B}}$ was computed such that for all $p \in \mathcal{L}_{L U B}^{\prime}$

$$
p \notin L_{\tilde{B}}+\mathbb{R}_{+}^{m}
$$

holds, where $\mathcal{L}_{L U B}^{\prime}$ is the list of local upper bounds at that node. Hence,

$$
p \notin f\left(\tilde{B}^{g, \mathbb{Z}}\right)+\mathbb{R}_{+}^{m} \text { for all } p \in \mathcal{L}_{L U B}^{\prime} .
$$

Let $\mathcal{L}_{L U B}$ be the final list of local upper bounds related to $\mathcal{L}_{P N S}$. By Lemma 3.3 a local upper bound $\hat{p} \in \mathcal{L}_{L U B}$ exists such that $z \leq \hat{p}$ holds, i.e., $\hat{p} \in f\left(\tilde{B}^{g, \mathbb{Z}}\right)+\mathbb{R}_{+}^{m}$. If $\hat{p} \in \mathcal{L}_{L U B}^{\prime}$ we directly get a contradiction to (6). Otherwise, from Remark 3.2, it follows that a local upper bound $\bar{p} \in \mathcal{L}_{L U B}^{\prime}$ exists such that $\hat{p} \leq \bar{p}$ holds. Hence, $\bar{p} \in f\left(\tilde{B}^{g, \mathbb{Z}}\right)+\mathbb{R}_{+}^{m}$ which contradicts $(6)$.

We now show that, in case MOMIX is applied, $\mathcal{L}_{P N S}$ is a "good" approximation of the nondominated set. This means that the distance of the image of efficient points from $\mathcal{L}_{P N S}$ is bounded by a quantity that depends on $\delta>0$, which is the input parameter of Algorithm 1. For this we exploit the Lipschitz continuity of the objective functions $f_{j}$, $j=1, \ldots, m$, which holds as the functions are continuously differentiable and the feasible sets are compact. Let $L_{j} \geq 0$ be the Lipschitz constant for function $f_{j}, j=1, \ldots, m$. 
Theorem 3.13 Let $\delta>0$ be the input parameter and $\mathcal{L}_{P N S}, \mathcal{L}_{\mathcal{S}}$ be the output of Algorithm 1 where MOMIX is applied, i.e., light $=0$. Let $\mathcal{L}_{L U B}$ be the local upper bound set with respect to $\mathcal{L}_{P N S}$ and $E \subseteq B^{g, \mathbb{Z}}$ be the efficient set of (MOMIC). Set $L=\max _{j=1, \ldots, m} L_{j}$. Then

$$
f(E) \subseteq\left(\bigcup_{p \in \mathcal{L}_{L U B}}\left(\{p\}-\mathbb{R}_{+}^{m}\right)\right) \bigcap\left(\bigcup_{z \in \mathcal{L}_{P N S}}\left(\{z-L \delta e\}+\mathbb{R}_{+}^{m}\right)\right)
$$

holds, where $e=(1, \ldots, 1)^{T} \in \mathbb{R}^{m}$.

Proof. Let $x \in E$. In order to prove that $f(x) \in \bigcup_{p \in \mathcal{L}_{L U B}}\left(\{p\}-\mathbb{R}_{+}^{m}\right)$ we distinguish two cases. Assume first that $f(x)$ belongs to the search region $S$ related to $\mathcal{L}_{P N S}$ (see Definition 3.1). Then, a local upper bound $p \in \mathcal{L}_{L U B}$ exists such that $f(x)$ belongs to the search zone $C(p)$ related to $p$. It follows that $f(x)<p$. On the other hand, if $f(x) \notin S$, by Definition 3.1 a point $z \in \mathcal{L}_{P N S}$ exists such that $z \leq f(x)$. Since $f(x)$ is nondominated and $z \in \mathcal{L}_{P N S}$ is the image of a feasible point, we necessarily have $f(x)=z$. From Lemma 3.3, a $p \in \mathcal{L}_{L U B}$ exists such that $f(x)=z \leq p$ holds.

Next, we prove $f(x) \in \bigcup_{z \in \mathcal{L}_{P N S}}\left(\{z-L \delta e\}+\mathbb{R}_{+}^{m}\right)$ : For this, we choose a box $\tilde{B} \in \mathcal{L}_{\mathcal{S}}$ with $x \in \tilde{B}^{g, \mathbb{Z}}$ which exists by Theorem 3.11. From Algorithm 2, if light $=0$, a feasible point $\hat{x} \in \tilde{B}^{g, \mathbb{Z}}$ is computed for $\tilde{B}$ (see line 24). The point $f(\hat{x})$ is an upper bound for (MOMIC) and then a candidate to belong to $\mathcal{L}_{P N S}$. Then, either $f(\hat{x})$ is an element of $\mathcal{L}_{P N S}$ or $z \in \mathcal{L}_{P N S}$ exists such that $z \leq f(\hat{x})$. Since $\omega(\tilde{B})<\delta$ holds, we have $\|x-\hat{x}\|<\delta$ and, by Lipschitz continuity of $f_{j}$ we obtain for all $j=1, \ldots, m$ : $\left|f_{j}(x)-f_{j}(\hat{x})\right| \leq L_{j} \delta \leq$ $L \delta, j=1, \ldots, m$. Therefore, since $L \delta \geq 0$, we have that $f_{j}(x) \geq f_{j}(\hat{x})-L \delta \geq z_{j}-L \delta$ for all $j=1, \ldots, m$ and the theorem is proved.

An illustration of Theorem 3.13 on an test instance solved by (MOMIC) is given in Figure 11 in Section 4.

\section{Numerical Results}

In this section, we present our numerical experiments on different instances of (MOMIC). In addition to some results on biobjective quadratic instances, we show results on an instance with $m=3$ and results on a mixed integer convex non-quadratic instance.

In our implementation of Algorithm 1 , at line 3 , in order to select a subbox $\tilde{B} \in \mathcal{L}_{\mathcal{W}}$, we consider the ideal point $f^{i d}$ computed according to (4). We pick at first those subboxes with the lexicographic smallest ideal point $f^{i d}$, with the idea that boxes with small $f^{i d}$ may lead to good upper bounds. Concerning the branching rule, we adopted two different strategies detailed in Section 4.1.

For solving the single-objective convex problems used to compute $f^{i d}$ and to define the hyperplanes $H^{\hat{\lambda}, p+\hat{t} e}$ we applied fmincon, the solver from the optimization toolbox of MATLAB. For all runs we set $\delta=0.1$ if it is not stated otherwise.

For the solution of the mixed integer convex programming problem used to define the hyperplane $H^{\hat{\lambda}, f(\hat{x})}$ that enriches the linear outer approximation of $f\left(\tilde{B}^{g, \mathbb{Z}}\right)$ (line 20 of Algorithm 2) we can adopt any solver which is able to deal with convex MINLPs as e.g. SCIP [22]. In our numerical experiments we mainly used quadratic instances and 
within our implementation of MOMIX we adopted the mixed integer quadratic solver of GUROBI [24].

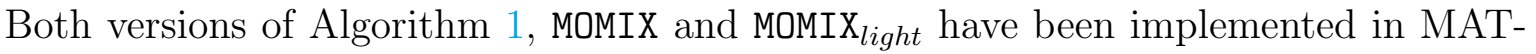
LAB R2018a. All experiments have been performed on a computer with Intel(R) Core(TM) i5-7400T CPU and 16 Gbytes RAM on operating system Windows 10 EnTERPRISE.

\subsection{Branching rules}

In our numerical experiments, we make use of two different branching rules. Both rules are based on the idea of partitioning boxes considering first the largest edges, giving priority to the integer variables in two different ways.

Let $\tilde{B}=[\tilde{l}, \tilde{u}]$ be a subbox of $B$. We consider the following two sets of indices in order to identify the branching variable $\hat{\imath} \in\{1, \ldots, n\}$ :

(br1) $J_{1}=\operatorname{argmax}\left\{\tilde{u}_{i}-\tilde{l}_{i} \mid i \in I\right\}$. If $\tilde{u}_{i}-\tilde{l}_{i}=0$ for all $i \in I$, i.e., in case all the integer variables are fixed, define $J_{1}=\operatorname{argmax}\left\{\tilde{u}_{i}-\tilde{l}_{i} \mid i \in\{1, \ldots, n\} \backslash I\right\}$. Choose $\hat{\imath} \in J_{1}$.

(br2) $J_{2}=\operatorname{argmax}\left\{\tilde{u}_{i}-\tilde{l}_{i} \mid i \in\{1, \ldots, n\}\right\}$. If $J_{2} \cap I \neq \emptyset$ holds, choose $\hat{\imath} \in J_{2} \cap I$. Otherwise, choose $\hat{\imath} \in J_{2}$.

The first strategy is standard in mixed integer procedures: the integer variables are fixed at first. The second strategy aims to reduce the largest edge of the boxes, no matter whether it is related to an integer variable or not. Only if there is more than one largest edges and one of them belongs to an integer variable, we prefer to branch at this variable. We will show that this second non-standard branching rule performs better on some of the test instances.

Once the branching variable $\hat{\imath} \in\{1, \ldots, n\}$ has been selected, we partition the box $\tilde{B}$ into two boxes $\tilde{B}_{1}, \tilde{B}_{2}$ as follows: We set for $c_{1}, c_{2} \in\left[\tilde{l}_{\hat{\imath}}, \tilde{u}_{\hat{\imath}}\right]$ :

$$
\tilde{B}_{1}:=\left[\tilde{l},\left(\tilde{u}_{1}, \ldots, \tilde{u}_{\hat{\imath}-1}, c_{1}, \tilde{u}_{\hat{\imath}+1}, \ldots, \tilde{u}_{n}\right)^{T}\right] \text { and } \tilde{B}_{2}:=\left[\left(\tilde{l}_{1}, \ldots, \tilde{l}_{\hat{\imath}-1}, c_{2}, \tilde{l}_{\hat{\imath}+1}, \ldots, \tilde{l}_{n}\right)^{T}, \tilde{u}\right] \text {. }
$$

Thereby, we differentiate between $\hat{\imath} \in I$ and $\hat{\imath} \notin I$. If $\hat{\imath} \notin I$, we set $c_{1}=c_{2}=\left(\tilde{l}_{\hat{\imath}}+\tilde{u}_{\hat{\imath}}\right) / 2$. If $\hat{\imath} \in I$, we set $c_{1}=\left\lfloor\left(\tilde{l}_{\hat{\imath}}+\tilde{u}_{\hat{\imath}}\right) / 2\right\rfloor$ and $c_{2}=\left\lceil\left(\tilde{l}_{\hat{\imath}}+\tilde{u}_{\hat{\imath}}\right) / 2\right\rceil$. In case $\tilde{l}_{\hat{\imath}}+\tilde{u}_{\hat{\imath}}$ is an even number, in order to avoid $\tilde{B}_{1} \cap \tilde{B}_{2} \neq \emptyset$, we split considering $c_{1}=\left(\tilde{l}_{\hat{\imath}}+\tilde{u}_{\hat{\imath}}\right) / 2$ and $c_{2}=1+\left(\tilde{l}_{\hat{\imath}}+\tilde{u}_{\hat{\imath}}\right) / 2$. Note that such a bisection excludes the infeasible part between $c_{1}$ and $c_{2}$.

As already mentioned in the introduction, we assume $B=[l, u] \subset \mathbb{R}^{n}$ with $l_{i}, u_{i} \in \mathbb{Z}$ for all $i \in I$. Then, for all subboxes $\tilde{B}$ obtained by any of the branching rules presented, it holds $\tilde{l}_{i}, \tilde{u}_{i} \in \mathbb{Z}$ for all $i \in I$. Furthermore, it is easy to see that both branching rules adopted in MOMIX and MOMIX light satisfy Assumption 3.10.

In order to clarify the differences between the two rules (br1) and (br2), we present the results obtained by MOMIX when applied to the following: 
Test instance 4.1 We study the biobjective mixed integer instance with two variables:

$$
\begin{array}{ll}
\min & \left(\begin{array}{l}
x_{1}+x_{2} \\
x_{1}^{2}+x_{2}^{2}
\end{array}\right) \\
\text { s.t. } & \left(x_{1}-2\right)^{2}+\left(x_{2}-2\right)^{2} \leq 36 \\
& x_{1} \in[-2,2] \\
& x_{2} \in[-4,4] \cap \mathbb{Z} .
\end{array}
$$

In Figure 6 and Figure 8, we show in gray the image of $B^{g, \mathbb{Z}}$ under the objective functions. In black we give the set $\mathcal{L}_{P N S}$ obtained by applying MOMIX with (br1) and (br2), respectively. Note that MOMIX is able to find in both cases a good approximation of the non-connected nondominated set of the instance. In Figure 5 and Figure 7, we report the partition of the box $B=\left[(-2,-4)^{T},(2,4)^{T}\right]$ obtained applying MOMIX with (br1) and (br2), respectively.

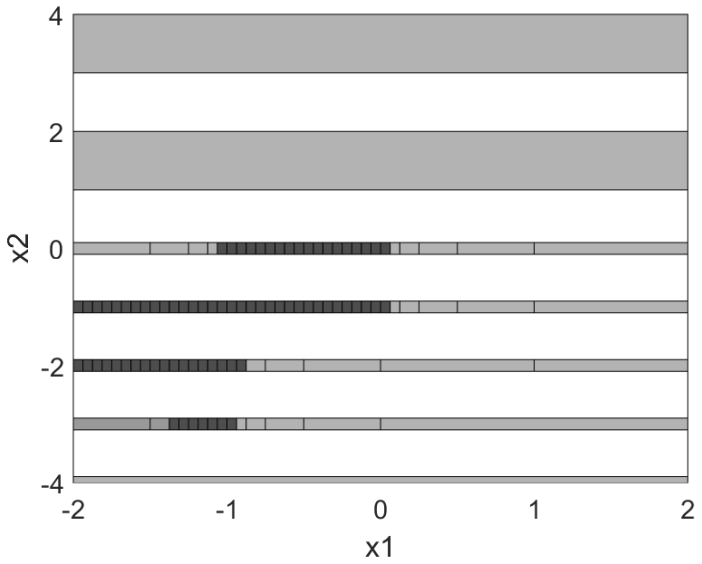

Figure 5: Partition of the box $B$ obtained by applying MOMIX with (br1)

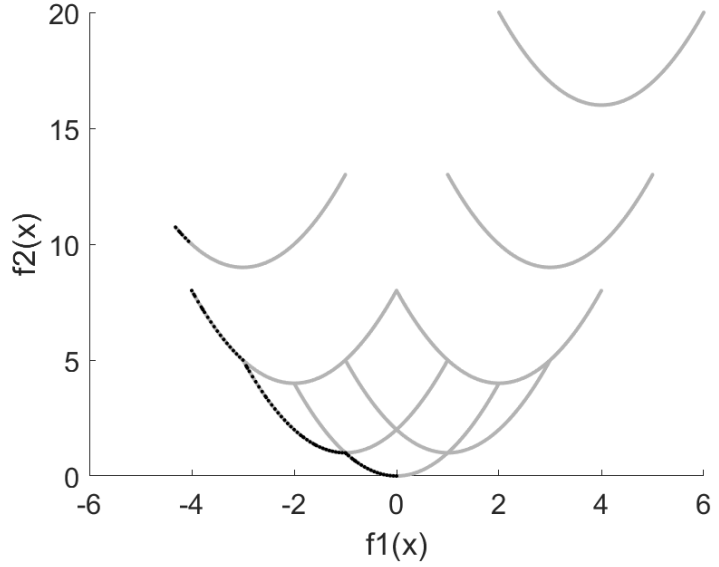

Figure 6: The set $\mathcal{L}_{P N S}$ obtained by applying MOMIX with (br1)

Both branching rules explore the whole feasible set of (T1). Even while they partition the box $B$ in different ways, the outputs of MOMIX are very similar, i.e., with (br1) and (br2) the boxes in the solution list $\mathcal{L}_{S}$ and the list of upper bounds $\mathcal{L}_{P N S}$ are nearly the same.

\subsection{Results on scalable instances}

In this section, we show results on three different test instances of (MOMIC), all scalable in the number of variables. We apply MOMIX and MOMIX $_{\text {light }}$ in combination with (br1) and (br2) on all instances. We analyze the impact of the branching rules as well as the difference between MOMIX and MOMIX light. Recall that MOMIX uses stronger lower bounds but these require to solve single-objective mixed integer convex programming problems. 


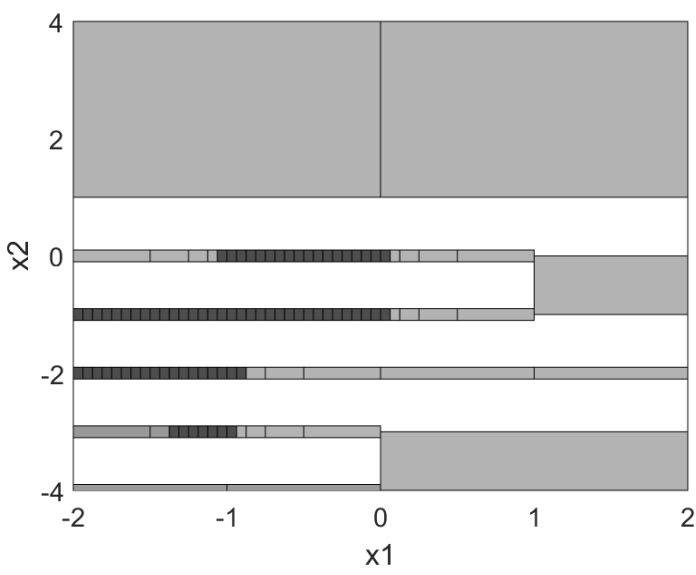

Figure 7: Partition of the box $B$ obtained by applying MOMIX with (br2)

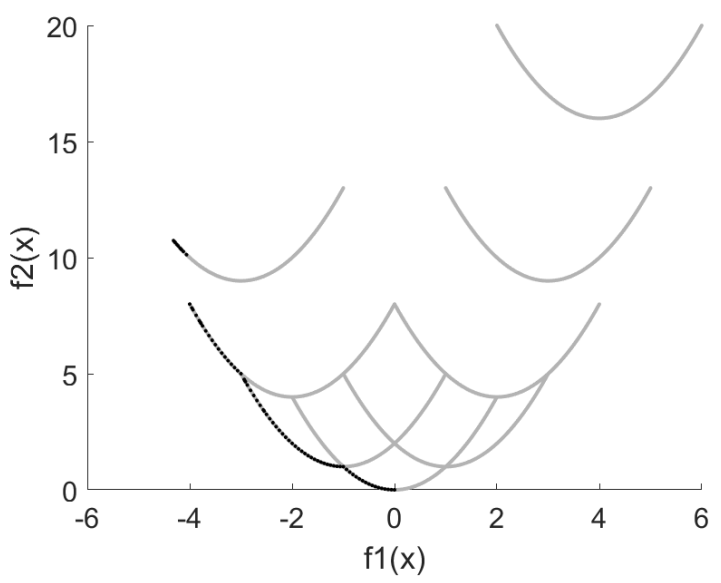

Figure 8: The set $\mathcal{L}_{P N S}$ obtained by applying MOMIX with (br2)

Test instance 4.2 This instance has quadratic objective functions. The number of integer variables can be set to different values. Let the matrices $Q_{1}$ and $Q_{2}$ be defined as follows:

$$
\left(Q_{1}\right)_{i, j}=\left\{\begin{array}{ll}
3 & \text { if } i=j=1 \\
4 & \text { if } i=j=n \\
1 & \text { else }
\end{array} \quad \text { and }\left(Q_{2}\right)_{i, j}= \begin{cases}2 & \text { if } i=j=1 \text { or } i=j=n \\
4 \quad \text { if } i=j \text { and } i \notin\{1, n\} \\
1 \quad \text { else. }\end{cases}\right.
$$

Then the optimization problem is stated by

$$
\begin{array}{ll}
\min & \left(\begin{array}{c}
x^{T} Q_{1}^{T} Q_{1} x+(1,2, \ldots, 2,1) x \\
x^{T} Q_{2}^{T} Q_{2} x+(-1,-2, \ldots,-2,5) x
\end{array}\right) \\
\text { s.t. } & x_{i} \in[-5,5], i \in\{1, \ldots, n\} \\
& I=\{3, \ldots, n\} .
\end{array}
$$

Note that $Q_{1}^{T} Q_{1}$ and $Q_{2}^{T} Q_{2}$ are positive semidefinite and hence $f_{1}$ and $f_{2}$ are convex functions.

Test instance 4.3 This instance is also scalable in the number of integer variables.

$$
\begin{array}{ll}
\min & \left(\begin{array}{c}
x_{1} \\
x_{2}+\sum_{i=3}^{n} 10\left(x_{i}-0.4\right)^{2}
\end{array}\right) \\
\text { s.t. } & \sum_{i=1}^{n} x_{i}^{2} \leq 4 \\
& x_{i} \in[-2,2] \text { for all } i=1, \ldots, n \\
& I=\{3, \ldots, n\}
\end{array}
$$

Here, we can explicitly give the set of all efficient points by

$$
E=\left\{x \in \mathbb{R}^{n} \mid x_{1}^{2}+x_{2}^{2}=4, x_{1} \in[-2,0], x_{2} \in[-2,0], x_{i}=0 \text { for all } i \geq 3\right\} .
$$


Test instance 4.4 In this instance both the number of continuous and integer variables, can be set to different values, with the restriction that $k^{c}=|\{1, \ldots, n\} \backslash I|$ has to be even.

$$
\begin{array}{ll}
\min & \left(\begin{array}{c}
\sum_{i=1}^{k^{c} / 2} x_{i}+\sum_{i=k^{c}+1}^{n} x_{i} \\
\sum_{i=k^{c} / 2+1}^{k^{c}} x_{i}-\sum_{i=k^{c}+1}^{n} x_{i}
\end{array}\right) \\
\text { s.t. } & \sum_{i=1}^{k^{c}} x_{i}^{2} \leq 1 \\
& x_{i} \in[-2,2] \text { for all } i=1, \ldots, n \\
& I=\left\{k^{c}+1, \ldots, n\right\}
\end{array}
$$

For both objective functions the Lipschitz constant is $L=\sqrt{k^{c} / 2+|I|}$.

For all instances but (T4) we set half an hour (1800 seconds) as time limit. For (T4) we set the time limit to one hour (3600 seconds).

In Figures 9, 10 and 11 we show our results in the image space. As the set $\mathcal{L}_{P N S}$ is similar for all versions of MOMIX and choices of the branching rule within one test instance, we present only the results for MOMIX with (br2) within the figures. In black we plot the points of $\mathcal{L}_{P N S}$. The gray points are the images of the feasible points, i.e., the upper bounds, computed along the algorithm. The parameter for the set from Theorem 3.13 applied to (T4) with $k^{c}=2$ and $|I|=1$ is $L \delta=0.1 \sqrt{2}$. Hence, the set described by $\bigcup_{z \in \mathcal{L}_{P N S}}\left(\{z-L \delta e\}+\mathbb{R}_{+}^{m}\right)$ is just a rough lower bound of the nondominated set. From a practical point of view, in all our test runs, the points from the lists $\mathcal{L}_{P N S}$ deliver a good approximation of the nondominated sets.

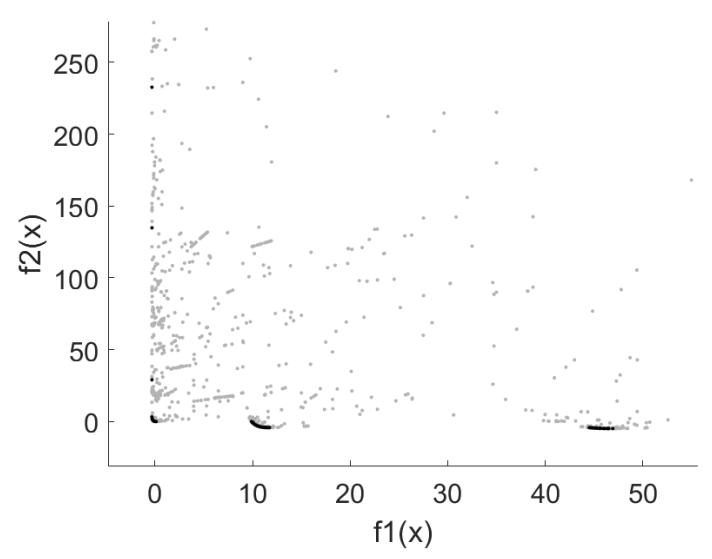

Figure 9: The set $\mathcal{L}_{P N S}$ of Instance (T2) for $|I|=5, n=7$.

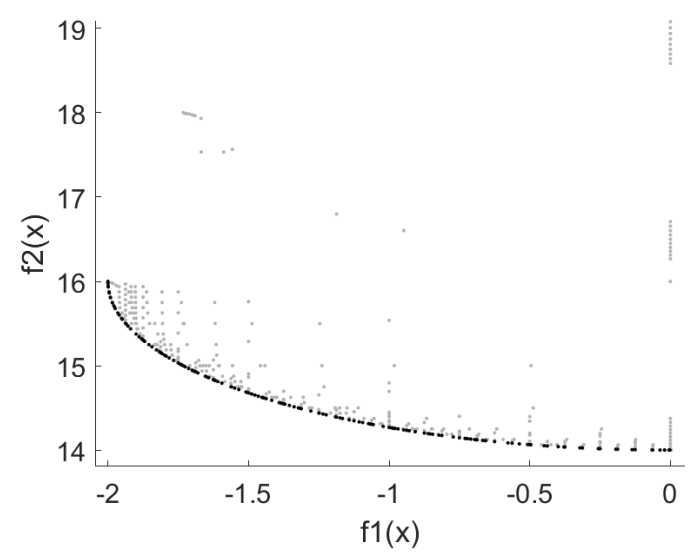

Figure 10: The set $\mathcal{L}_{P N S}$ of Instance (T3) for $|I|=10, n=12$.

The numerical results on all instances are shown in Table 1. In the first two columns we report the number of integer $(|I|)$ and the number of continuous variables $(|C|)$ for

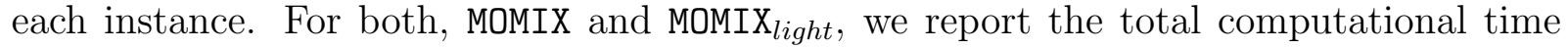
in seconds (CPU) and the number of considered boxes in the branching tree (\#nod). 

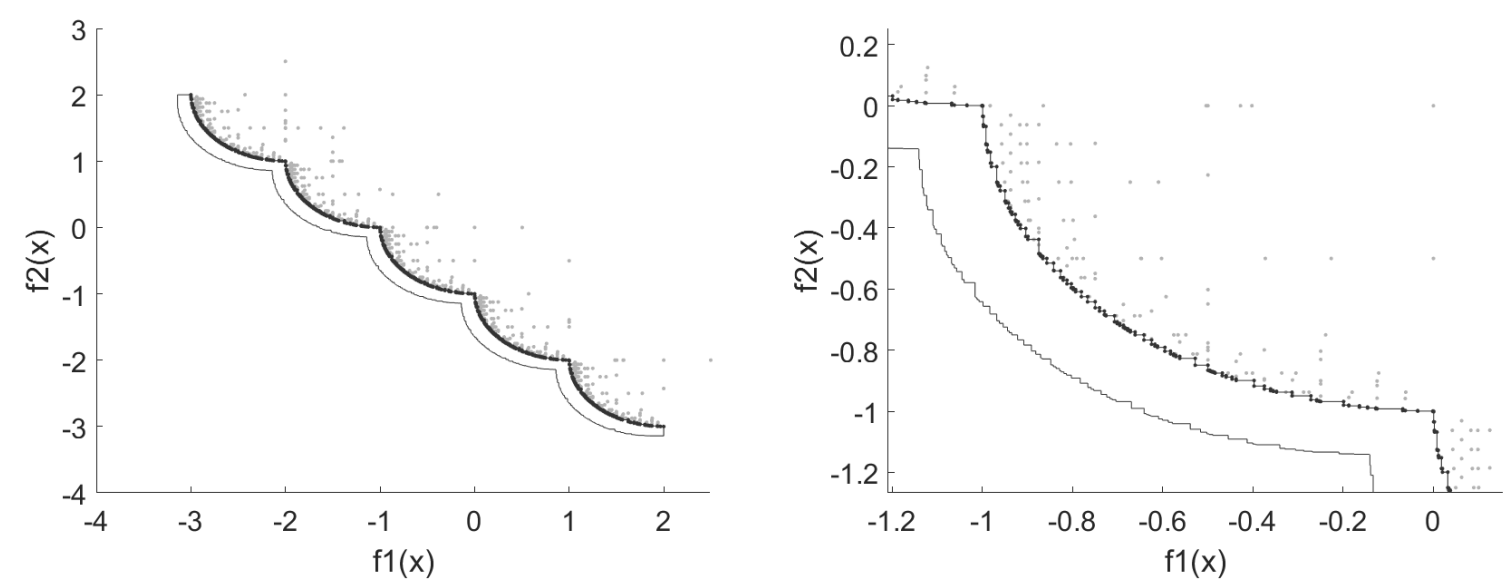

Figure 11: The set $\mathcal{L}_{P N S}$ of Instance (T4) for $|I|=1, n-|I|=2$ and the boundary of the set from Theorem $3.13, L \delta=0.1 \sqrt{2}$. Right picture shows a detail of the left one.

For MOMIX we additionally report the total time needed by Gurobi to address the singleobjective mixed integer quadratic problems (MIQP). Failures, i.e., instances for which the time limit was exceeded, are marked with "-".

\begin{tabular}{|c|c|c|c|c|c|c|c|c|c|c|c|}
\hline & & \multicolumn{6}{|c|}{ MOMIX } & \multicolumn{4}{|c|}{ MOMIX $_{\text {light }}$} \\
\hline & & \multicolumn{3}{|c|}{ (br1) } & \multicolumn{3}{|c|}{ (br2) } & \multicolumn{2}{|c|}{ (br1) } & \multicolumn{2}{|c|}{ (br2) } \\
\hline$|I|$ & $|C|$ & $\mathrm{CPU}$ & \#nod & MIQP & $\mathrm{CPU}$ & \#nod & MIQP & CPU & $\#$ nod & $\mathrm{CPU}$ & \#nod \\
\hline \multicolumn{12}{|c|}{ Test instance (T2) - time limit 1800s } \\
\hline 1 & 2 & 40.1 & 757 & 2.3 & 38.7 & 765 & 2.3 & 849.9 & 609 & 524.5 & 669 \\
\hline 2 & 2 & 30.8 & 537 & 1.6 & 31.6 & 575 & 1.7 & 667.2 & 555 & 563.0 & 641 \\
\hline 3 & 2 & 31.0 & 535 & 1.5 & 30.8 & 521 & 1.5 & 1381.2 & 1127 & 814.4 & 917 \\
\hline 4 & 2 & 34.7 & 567 & 1.7 & 65.6 & 1095 & 3.0 & - & - & 1134.9 & 1285 \\
\hline 5 & 2 & 38.5 & 587 & 1.6 & 81.5 & 1259 & 3.2 & - & - & - & - \\
\hline 10 & 2 & 350.3 & 2707 & 9.5 & - & - & - & - & - & - & - \\
\hline \multicolumn{12}{|c|}{ Test instance (T3) - time limit 1800s } \\
\hline 1 & 2 & 15.5 & 301 & 0.5 & 14.6 & 299 & 0.4 & 1045.4 & 299 & 1025.6 & 299 \\
\hline 10 & 2 & 36.5 & 413 & 1.2 & 27.1 & 353 & 0.7 & - & - & - & - \\
\hline 20 & 2 & - & - & - & 46.9 & 411 & 0.9 & - & - & - & - \\
\hline 30 & 2 & - & - & - & 80.4 & 471 & 1.1 & - & - & - & - \\
\hline 50 & 2 & - & - & - & - & - & - & - & - & - & - \\
\hline \multicolumn{12}{|c|}{ Test instance (T4) - time limit 3600s } \\
\hline 1 & 2 & 41.5 & 749 & 1.3 & 44.3 & 771 & 1.3 & 296.3 & 747 & 225.6 & 801 \\
\hline 2 & 2 & 226.2 & 3683 & 6.3 & 240.5 & 3761 & 6.2 & - & - & 3090.4 & 3701 \\
\hline 3 & 2 & 1354.9 & 19127 & 32.3 & 1321.5 & 18451 & 31.1 & - & - & - & - \\
\hline 1 & 4 & 2199.5 & 23935 & 53.5 & 2246.6 & 24399 & 53.8 & - & - & - & - \\
\hline
\end{tabular}

Table 1: Numerical results for test instances (T2), (T3) and (T4)

We observe that MOMIX outperforms MOMIX $_{\text {light }}$ on all test instances. MOMIX is able to solve a higher number of instances within the time limit. This seems to indicate that the improved lower bounding procedure of MOMIX and the effort in solving singleobjective mixed integer convex problems pays off. We notice that the time Gurobi needs to address the single-objective mixed integer subproblems is a small percentage of the whole computational time.

Regarding the two branching rules, we can notice some differences as soon as the

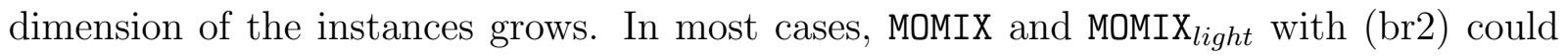
solve instances with a larger number of integer variables. 
By using the MATLAB profiler on our code we got that the bottleneck in our implementation is fmincon: Most of the CPU time was spent to solve the single-objective continuous convex problems. Note that fmincon can be replaced by any solver for convex problems within both MOMIX and MOMIX light.

In the following, we compare MOMIX with the $\varepsilon$-constraint method for biobjective mixed integer quadratic optimization on a specific instance. The $\varepsilon$-constraint method minimizes parameter-dependent single-objective optimization problems of the following form:

$$
\begin{array}{cl}
\min & f_{2}(x) \\
\text { s.t. } & f_{1}(x) \leq \varepsilon \\
& x \in B^{g, \mathbb{Z}} .
\end{array}
$$

In the biobjective case, the minima of the functions $f_{1}$ and $f_{2}$ define the interval where the parameter $\varepsilon$ belongs. In our case, the parameter $\varepsilon$ varies between $\min \left\{f_{1}(x) \mid x \in B^{g, \mathbb{Z}}\right\}$ and $f_{1}(\hat{x})$ with $\hat{x} \in \operatorname{argmin}\left\{f_{2}(x) \mid x \in B^{g, \mathbb{Z}}\right\}$. For a step size $\delta>0$, the parameter $\varepsilon$ can now be chosen equidistantly within that interval by setting $\varepsilon_{1}:=f_{1}(\hat{x})$, and $\varepsilon_{k+1}:=\varepsilon_{k}-\delta$ for $k \geq 1$. For a better handling of gaps, as they appear for instance in test instance (T2), one can also adapt the choice of the parameters by setting $\varepsilon_{k+1}:=f_{1}\left(x^{k}\right)-\delta$ where $x^{k}$ denotes a minimal solution of $\left(\mathrm{P}_{\varepsilon_{k}}\right)$. For both approaches one typically initializes solving $\left(\mathrm{P}_{\varepsilon_{k+1}}\right)$ with $x^{k}$ as starting point.

We show in Figure 12 the comparison of MOMIX with the $\varepsilon$-constraint method with adaptive parameter choiceon the Instance (T2) with $|I|=5, n=7$ and $\delta=0.1$. The points of $\mathcal{L}_{P N S}$ are black points, while the solutions of the $\varepsilon$-constraint method are diamonds $(\diamond)$.

The $\varepsilon$-constraint method with equidistant parameters solved in total 475 single-objective mixed integer problems within 5.2 seconds. However, it only computed 52 different solutions, meaning that 423 solved optimization problems did not deliver a new solution. This could be strongly improved by an adaptive parameter choice which resulted in 50 single-objective mixed integer problems within 0.7 seconds and 50 different solutions.

Note that the approximation of the nondominated set differs. It can be seen that the first part of the nondominated set (see second picture of Figure 12) is much better approximated by the solutions of MOMIX. Regarding the other parts of the nondominated set they are similarly approximated by both MOMIX and the adaptive $\varepsilon$-constraint method (see third and fourth picture of Figure 12). The choice of the parameters $\varepsilon$ gets more difficult in higher dimensions. Moreover, one does not get covering results as those presented in Theorem 3.11 or Theorem 3.13.

\subsection{Results on a triobjective instance}

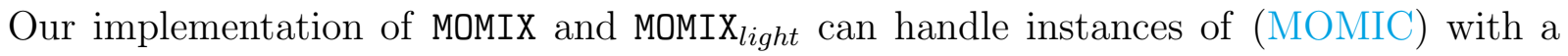
general number of objective functions $m \geq 2$. In the following, we present the results obtained by applying MOMIX with branching rule (br2). 

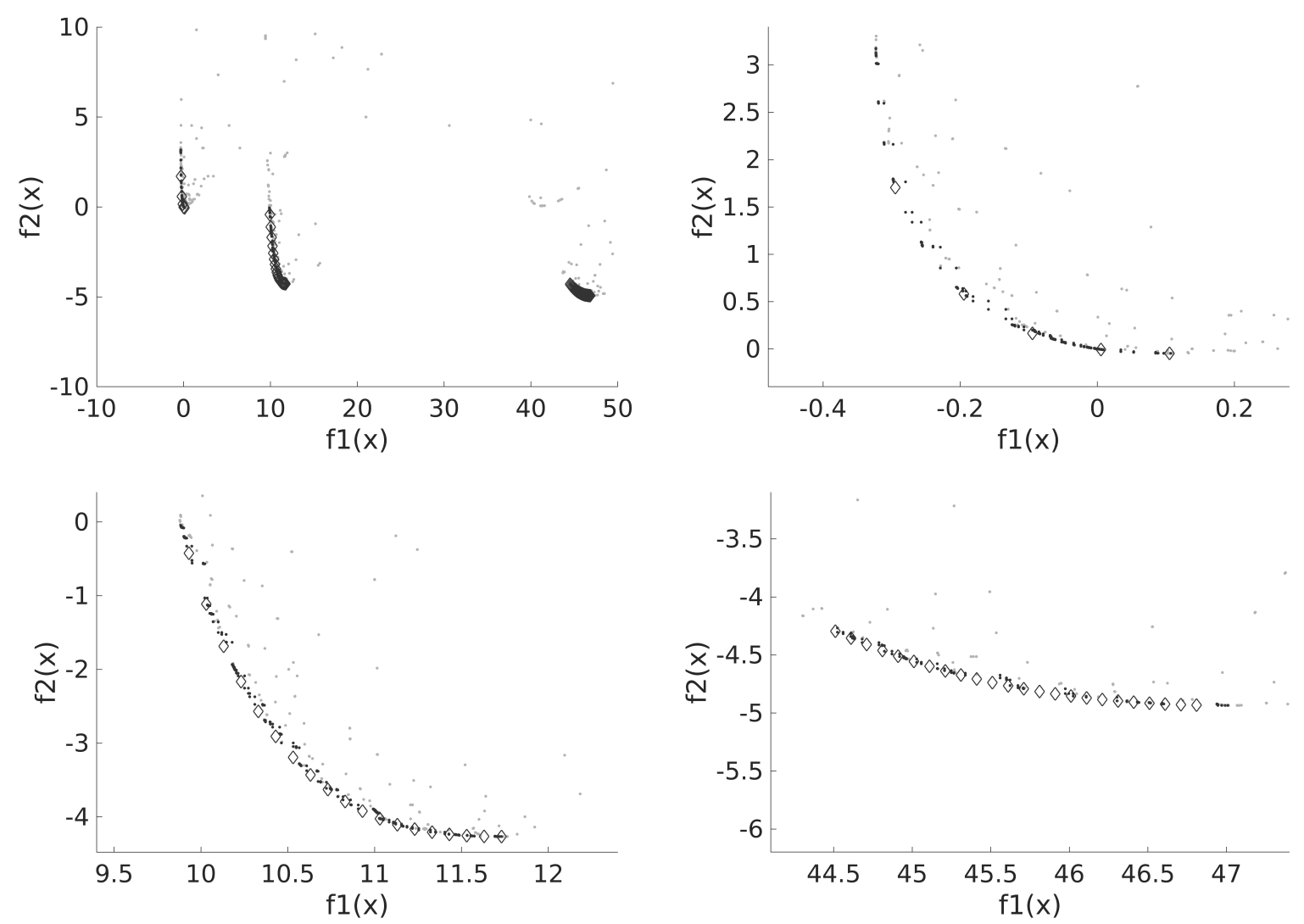

Figure 12: Instance (T2) with $|I|=5, n=7$ : The set $\mathcal{L}_{P N S}$ in comparison with the solutions of a $\varepsilon$-constraint method with adaptive parameter choice $(\diamond)$. The last three pictures show each part of the nondominated set separately.

Test instance 4.5 We consider the triobjective mixed integer instance

$$
\begin{array}{ll}
\min & \left(\begin{array}{l}
x_{1}+x_{4} \\
x_{2}-x_{4} \\
x_{3}+x_{4}^{2}
\end{array}\right) \\
\text { s.t. } & \sum_{i=1}^{3} x_{i}^{2} \leq 1 \\
& x_{i} \in[-2,2] \text { for all } i=1, \ldots, 4 \\
& x_{4} \in \mathbb{Z} .
\end{array}
$$

We set $\delta=0.5$ in MOMIX. In order to detect $\mathcal{L}_{\mathcal{S}}$, the cover of the efficient set of Problem (T5), MOMIX needed to explore 1237 nodes. This was done within 190 seconds CPU time.

In Figure 13 the points in $\mathcal{L}_{P N S}$ are plotted in black, giving an approximation of the nondominated set of Problem (T5). In gray we plot the images of the feasible points computed along the algorithm. 

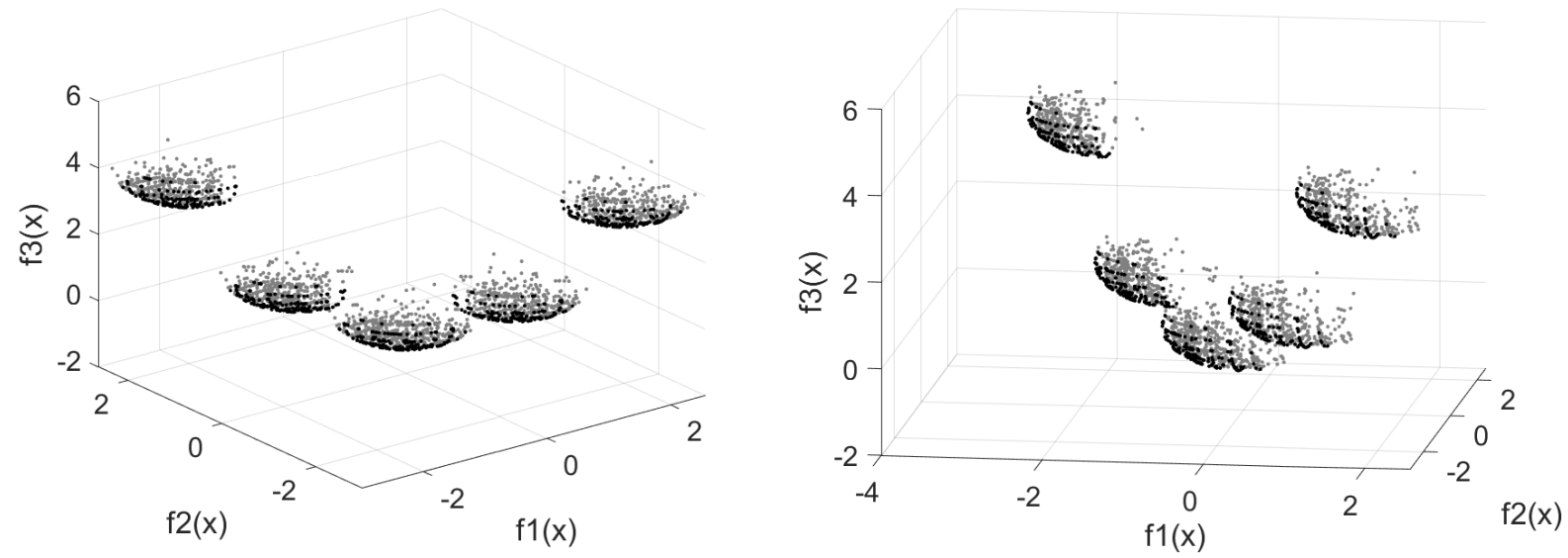

Figure 13: The set $\mathcal{L}_{P N S}$ for Problem (T5) from two different perspectives.

\subsection{Results on a non-quadratic convex instance}

As a further example, we report the results obtained applying MOMIX $_{\text {light }}$ with branching rule (br1) on the following non-quadratic instance:

Test instance 4.6

$$
\begin{array}{ll}
\min & \left(\begin{array}{c}
x_{1}+x_{3} \\
x_{2}+\exp \left(-x_{3}\right)
\end{array}\right) \\
\text { s.t. } & x_{1}^{2}+x_{2}^{2} \leq 1 \\
& x_{i} \in[-2,2] \text { for all } i=1, \ldots, 3 \\
& x_{3} \in \mathbb{Z}
\end{array}
$$

Note that the second objective of Problem (T6) is a convex non-quadratic function.

As already mentioned at the beginning of the section, in our implementation of MOMIX we use GUROBI [24] as mixed integer quadratic solver and we did not include any other solver within it. Therefore, in order to solve Problem (T6) we applied MOMIX light $_{\text {setting }}$ $\delta=0.1$. MOMIX light was able to detect $\mathcal{L}_{\mathcal{S}}$ by addressing 1105 nodes within 20 seconds CPU time. In Figure 14, we plot the obtained approximation of the nondominated set of Problem (T6).

\section{Conclusions}

In this paper we devised the first non-scalarization based deterministic algorithm for solving multiobjective mixed integer convex programming problems. The method is based on linear outer approximations of the image set. We first build linear outer approximations of the convex relaxation of the problem by adaptively computing hyperplanes considering some meaningful local upper bounds. Then, in case we want to improve our lower bound, we compute additional hyperplanes that outer approximate the convex hull of the true image set. This is again done in an adaptive way, taking specific local upper bounds into 


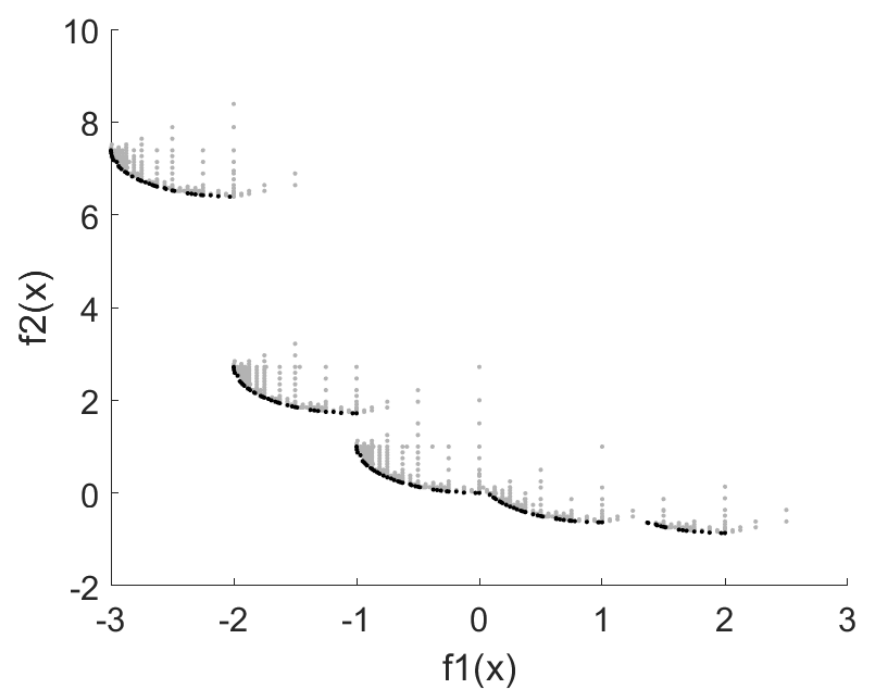

Figure 14: The set $\mathcal{L}_{P N S}$ of Problem (T6) obtained by MOMIX

account. The local upper bound sets are updated as soon as a new upper bound is found and are used both to have a pruning criterion and to approximate the dominated set. Theoretical results related to the correctness of our algorithm are provided. Numerical examples on both, biobjective and triobjective, instances show the ability of our procedure to detect nondominated points of multiobjective mixed integer convex programming problems. We also explored the possibility of using two different branching rules.

\section{Acknowledgments}

The authors wish to thank the two anonymous referees for their valuable comments and remarks which helped us to improve the paper. The first author acknowledges support within the DAAD scholarship No 57440915. She further acknowledges support within the project No RP1181641D22304F which has received funding from Sapienza, University of Rome. The third author thanks the Carl-Zeiss-Stiftung and the DFG-founded Research Training Group 1567 for financial support. The current work of the fourth author is funded by the Deutsche Forschungsgemeinschaft under project ID 392195690.

\section{References}

[1] Nathan Adelgren and Akshay Gupte. Branch-and-bound for biobjective mixed integer programming. arXiv preprint arXiv:1709.03668, 2017.

[2] P. Belotti, C. Kirches, S. Leyffer, J. Linderoth, J. Luedtke, and A. Mahajan. Mixedinteger nonlinear optimization. Acta Numerica, 22:1-131, 2013. 
[3] N. Boland, H. Charkhgard, and M. Savelsbergh. A criterion space search algorithm for biobjective integer programming: The balanced box method. INFORMS J. Comput., 27(4):735-754, 2015.

[4] N. Boland, H. Charkhgard, and M. Savelsbergh. The L-shape search method for triobjective integer programming. Math. Program. Comput., 8(2):217-251, 2016.

[5] N. Boland, H. Charkhgard, and M. Savelsbergh. A new method for optimizing a linear function over the efficient set of a multiobjective integer program. Eur. J. Oper. Res., 260(3):904-919, 2017.

[6] N. Boland, H. Charkhgard, and M. Savelsbergh. The quadrant shrinking method: A simple and efficient algorithm for solving tri-objective integer programs. Eur. J. Oper. Res., 260(3):873-885, 2017.

[7] N. Boland, A. C. Eberhard, F. Engineer, and A. Tsoukalas. A new approach to the feasibility pump in mixed integer programming. SIAM J. Optim., 22(3):831-861, 2012.

[8] Natashia Boland, Hadi Charkhgard, and Martin Savelsbergh. The triangle splitting method for biobjective mixed integer programming. In Jon Lee and Jens Vygen, editors, Integer Programming and Combinatorial Optimization, pages 162-173. Springer International Publishing, 2014.

[9] Natashia Boland, Hadi Charkhgard, and Martin Savelsbergh. A criterion space search algorithm for biobjective mixed integer programming: The triangle splitting method. INFORMS Journal on Computing, 27(4):597-808, 2015.

[10] P. Bonami, G. Cornuéjols, A. Lodi, and F. Margot. A feasibility pump for mixed integer nonlinear programs. Math. Program., 119(2):331-352, 2009.

[11] R. S. Burachik, C. Y. Kaya, and M. M. Rizvi. Algorithms for generating pareto fronts of multi-objective integer and mixed-integer programming problems. arXiv:1903.07041v1.

[12] R. S. Burachik, C. Y. Kaya, and M. M. Rizvi. A new scalarization technique to approximate pareto fronts of problems with disconnected feasible sets. J. Optim. Thery Appl., 162(2):428-446, 2014.

[13] R. S. Burachik, C. Y. Kaya, and M. M. Rizvi. A new scalarization technique and new algorithms to generate pareto fronts. SIAM J. Optim., 27(2):1010-1034, 2017.

[14] V. Cacchiani and C. D'Ambrosio. A branch-and-bound based heuristic algorithm for convex multi-objective MINLPs. Eur. J. Oper. Res., 260(3):920-933, 2017.

[15] M. De Santis, S. Lucidi, and F. Rinaldi. A new class of functions for measuring solution integrality in the feasibility pump approach. SIAM J. Optim., 23(3):15751606, 2013. 
[16] M. Ehrgott and X. Gandibleux. Bound sets for biobjective combinatorial optimization problems. Comput. Oper. Res., 34:2674-2694, 2007.

[17] M. Ehrgott, L. Shao, and A. Schöbel. An approximation algorithm for convex multiobjective programming problems. J. Global Optim., 50(3):397-416, 2011.

[18] M. Ehrgott, C. Waters, R. Kasimbeyli, and O. Ustun. Multiobjective programming and multiattribute utility functions in portfolio optimization. INFOR Inf. Syst. Oper. Res., 47(1):31-42, 2009.

[19] Ali Fattahi and Metin Turkay. A one direction search method to find the exact nondominated frontier of biobjective mixed-binary linear programming problems. European Journal of Operational Research, 266(2):415-425, 2018.

[20] M. Fischetti, F. Glover, and A. Lodi. The feasibility pump. Math. Program., 104(1):91-104, 2005.

[21] B. Geißler, A. Morsi, L. Schewe, and M. Schmidt. Penalty alternating direction methods for mixed-integer optimization: A new view on feasibility pumps. SIAM J. Optim., 27(3):1611-1636, 2017.

[22] A. Gleixner, M. Bastubbe, L. Eifler, T. Gally, G. Gamrath, R. L. Gottwald, G. Hendel, C. Hojny, T. Koch, M. E. Lübbecke, S. J. Maher, M. Miltenberger, B. Müller, M. E. Pfetsch, C. Puchert, D. Rehfeldt, F. Schlösser, C. Schubert, F. Serrano, Y. Shinano, J. M. Viernickel, M. Walter, F. Wegscheider, J. T. Witt, and J. Witzig. The SCIP Optimization Suite 6.0. ZIB-Report 18-26, Zuse Institute Berlin, July 2018.

[23] O. Günlük, J. Lee, and R. Weismantel. Minlp strengthening for separable convex quadratic transportation-cost ufl. IBM Res. Report, pages 1-16, 2007.

[24] LLC Gurobi Optimization. Gurobi optimizer reference manual, 2018.

[25] J. Jahn. Vector Optimization. Springer, 2009.

[26] K. Klamroth, R. Lacour, and D. Vanderpooten. On the representation of the search region in multi-objective optimization. Eur. J. Oper. Res., 245(3):767-778, 2015.

[27] A. Löhne, B. Rudloff, and F. Ulus. Primal and dual approximation algorithms for convex vector optimization problems. J. Global Optim., 60(4):713-736, 2014.

[28] G. Mavrotas and D. Diakoulaki. A branch and bound algorithm for mixed zero-one multiple objective linear programming. Eur. J. Oper. Res., 107(3):530-541, 1998.

[29] G. Mavrotas and D. Diakoulaki. Multi-criteria branch and bound: A vector maximization algorithm for mixed 0-1 multiple objective linear programming. Appl. Math. Comput., 171(1):53-71, 2005.

[30] J. Niebling and G. Eichfelder. A branch-and-bound-based algorithm for nonconvex multiobjective optimization. SIAM J. Optim., 29(1):794-821, 2019. 
[31] Julia Niebling and Gabriele Eichfelder. A branch-and-bound algorithm for biobjective problems. In Proceedings of the XIII Global Optimization Workshop GOW16, pages 57-60, 2016.

[32] Y. Peng and L. Yu. Multiple criteria decision making in emergency management. Comput. Oper. Res., 42:1-2, 2014.

[33] Tyler Perini, Natashia Boland, Diego Pecin, and Martin Savelsbergh. A criterion space method for biobjective mixed integer programming: The boxed line method. INFORMS J. Comput., 2019.

[34] S.A.B. Rasmi, A. Fattahi, and M. Türkay. Sass: slicing with adaptive steps search method for finding the non-dominated points of tri-objective mixed-integer linear programming problems. Ann. Oper. Res., 2019.

[35] Stefan Rocktäschel. A Branch-and-Bound Algorithm for Multiobjective Mixed-integer Convex Optimization. Best Masters Book Series. Springer, 2020.

[36] Payman Ghasemi Saghand, Hadi Charkhgard, and Changhyun Kwon. A branch-andbound algorithm for a class of mixed integer linear maximum multiplicative programs: A bi-objective optimization approach. Comput. Oper. Res., 101:263-274, 2019.

[37] F. Sourd and O. Spanjaard. A multiobjective branch-and-bound framework: Application to the biobjective spanning tree problem. INFORMS J. Comput., 20(3):472-484, 2008.

[38] Banu Soylu and Gazi Bilal Yldz. An exact algorithm for biobjective mixed integer linear programming problems. Computers $\&$ Operations Research, 72:204-213, 2016.

[39] P. Xidonas, G. Mavrotas, and J. Psarras. Equity portfolio construction and selection using multiobjective mathematical programming. J. Global Optim., 47(2):185-209, 2010. 